Submitted to the Astrophysical Journal

Preprint typeset using $\mathrm{IAT}_{\mathrm{E} X}$ style emulateapj v. 5/25/10

\title{
IMPLICATIONS OF X-RAY LINE VARIATIONS FOR 4U1822-371
}

\author{
L. Ji ${ }^{1}$, N. S. Schulz ${ }^{1}$, M. A. NowaK ${ }^{1}$, C. R. Canizares ${ }^{1}$, \\ Submitted to the Astrophysical Journal
}

\begin{abstract}
$4 \mathrm{U} 1822-371$ is one of the proto-type accretion disk coronal sources with an orbital period of about 5.6 hours. The binary is viewed almost edge-on at a high inclination angle of 83 degrees, which makes it a unique candidate to study binary orbital and accretion disk dynamics in high powered X-ray sources. We observed the X-ray source in 4U 1822-371 with the Chandra High Energy Transmission Grating Spectrometer (HETGS) for almost nine binary orbits. X-ray eclipse times provide an update of the orbital ephemeris. We find that our result follows the quadratic function implied by previous observations; however, it suggests a flatter trend. Detailed line dynamics also confirm a previous suggestion that the observed photo-ionized line emission originates from a confined region in the outer edge of the accretion disk near the hot spot. Line properties allow us to impose limits on the size of accretion disk, the central corona, and the emission region. The photo-ionized plasma is consistent with ionization parameters of $\log \xi>2$, and when combined with disk size and reasonable assumptions for the plasma density, this suggests illuminating disk luminosities which are over an order of magnitude higher than what is actually observed. That is, we do not directly observe the central emitting X-ray source. The spectral continua are best fit by a flat power law with a high energy cut-off and partial covering absorption $\left(N_{\mathrm{H}}\right.$ ranging from $\left.5.4-6.3 \times 10^{22} \mathrm{~cm}^{-2}\right)$ with a covering fraction of about $50 \%$. We discuss some implications of our findings with respect to the photo-ionized line emission for the basic properties of the X-ray source.
\end{abstract}

Subject headings: accretion, accretion disks — binaries: eclipsing — stars: individual (4U 1822-371) — X-rays: binaries

\section{INTRODUCTION}

Eclipsing X-ray binaries are still fairly rare as they require a view of the accretion disk that is within several degrees of the edge. The low-mass X-ray binary (LMXB) $4 \mathrm{U} 1822-371$ is a specifically rare object because it also has a relatively short orbital period which produces eclipses every $5.57 \mathrm{~h}$ and a light curve which is very sensitive to the structure of the accretion disk rim (White \& Holt 1982). Repetitive features in the light curve as well as the fact that the eclipse is only partial provide some direct geometrical constraints such as a viewing angle $i$ within $75^{\circ}$ and $85^{\circ}$ as well as a disk radius of $(5-7.3) \times 10^{10} \mathrm{~cm}$ for this range of viewing angles (Mason \& Cordova 1982; Hellier \& Mason 1989). Heinz \& Nowak (2001) determined the most accurate inclination of $i=82.5 \pm 1.5$ from Rossi X-Ray Timing Explorer (RXTE) data.

Its generally hard X-ray spectrum is an indicator that there is an accretion disk corona (ADC) in the line of sight to the central X-ray source which also partially obscures the source with a radial extent of $\sim 3 \times 10^{10}$ cm (White et al. 1981; White \& Holt 1982). X-ray spectra from the pre-Chandra era were complex to model as they seem to require additional and unresolved soft emission line structures in addition to a significant Fe K fluorescence line. This made 4U 1822-371 the archetypical example of a LMXB ADC source (White et al. 1981, 1997; Parmar et al. 2000). The first Chandra observation in 2000 finally resolved these line structures into discrete emission lines from photo-ionized $\mathrm{O}, \mathrm{Ne}, \mathrm{Mg}$, $\mathrm{Si}, \mathrm{S}$, and Fe ions (Cottam et al. 2001). Phase-resolved

\footnotetext{
${ }^{1}$ MIT Kavli Institute for Astrophysics \& Space Research
}

spectra also suggested that the line emission originates from a highly confined area, likely the illuminated hot spot. From the persistence of the Fe K fluorescence line throughout the binary orbit, Cottam et al. (2001) also concluded that its emission must come from a more extended region around the disk. A recent survey of all Xray binaries in the Chandra archive indeed shows that in nearly all cases narrow line fluorescence originates from more or less spherically distributed material around the centrally illuminating source (Torrejon, et al. 2010).

More detailed dynamical information in LMXBs are hard to come by simply because a clear identification of the low-mass companion at distances of several kpc is usually impossible. Jonker \& van der Klis (2001) detected $0.59 \mathrm{~s}$ pulsations from the neutron star in $4 \mathrm{U}$ 1822-371 and determined a donor star mass of 0.4 $M_{\odot} \quad$ assuming a neutron star mass of $1.4 M_{\odot}$. From pulsation time delays they also determined that the orbit must be circular. By observing Bowen fluorescence from the X-ray heated face of the companion star, Casares et al. (2003) determined a radial velocity semiamplitude of $300 \mathrm{~km} \mathrm{~s}^{-1}$, which yielded a companion mass of $0.36 M_{\odot}$. However Cowley et al. (2003) used strong He I absorption to measure a lower limit of the orbital velocity of the donor star to $234 \mathrm{~km} \mathrm{~s}^{-1}$, consistent with previous measurements (Harlaftis et al. 1997), providing a lower limit to the donor mass of again 0.4 $M_{\odot}$. In addition, Cowley et al. (2003) also measured $\mathrm{He}$ II and $\mathrm{H} \alpha$ emission line velocities and determined a systemic velocity of $-103 \mathrm{~km} \mathrm{~s}^{-1}$. They argued that such a large negative value in direction of the Galactic center might indicate that $4 \mathrm{U} 1822-371$ is either part of the halo population or it was given a significant kick when 
the neutron star was formed.

In this paper we use phase- and highly spectrally resolved X-ray spectra over almost nine binary orbits in order to further investigate the origin of the recombination line emission and use their dynamics to diagnose properties of the accretion disk.

\section{DATA REDUCTION}

We observed the source $4 \mathrm{U} 1822-371$ on 2008 May 20 (ID9076) and 23 (ID9858) with the Chandra HETGS (Canizares et al. 2000) in a standard configuration via the HETG Guaranteed Time Observation program (see table 1). With a total exposure time approximately $150 \mathrm{ksec}$, these two observations cover seven (three and four, respectively) full binary periods. We reprocess all the observations using CIAO Version 4.2 with the most recent CALDB products.

For each observation, we redetermine the positions of the zeroth-order centroid using the $\mathrm{f}$ indzo script for optimal wavelength accuracy. We extract all first-order spectra in HEG and MEG and co-add them into one single spectrum. This has the consequence that we adopt a spectral resolution near $0.021 \mathrm{~m} \AA$ provided by the medium energy transmission grating grid throughout the entire wavelength band. The sacrificed resolution is a trade-off for easier handling of the spectra with respect to signal-to-noise ratios, specifically for the phase-resolved spectral analysis. Light curves and spectral analysis are based on ISIS Versions 1.5.0-1.6.1 (Houck \& Denicola 2000).

\section{ANALYSIS AND RESULTS}

Eclipses are rare in LMXBs. They, however, contain critical dynamical information with respect to the geometry of the disk and its environment. A broad eclipse, for example, would indicate an extended companion. A partial eclipse may indicate the presence of large scale scattering structures such as ADC. In a first step in the analysis we derive an improved ephemeris based on all three HETGS observations and test if the orbital period is changing. A second step investigates orbital phase variations of bright X-ray lines. In a third step we fit a phase-binned spectrum at an orbital phase which allows a direct view of the photo-ionized region with appropriate continuum and photo-ionization models.

\subsection{An Ephemeris Update}

All existing HETGS observations of 4U 1822-371 yield a total of 9 complete eclipse light curves. The year 2000 data indicated a slowly increasing orbital period. Here we first folded each of the observations into phase bins using a fixed period as determined by Parmar et al. (2000). Then we fitted each phase-folded light curve with Gaussian and sinusoidal functions. The results are shown in the right upper panel of Figure 1 for ID9858 as an illustration. The new eclipse time can be well-determined. Once we join this new eclipse time to the already published X-ray eclipse times (Parmar et al. 2000, and the reference therein), we can fit the timing residuals with respect to the best-fit linear ephemeris $\left(\chi^{2}=35.99\right.$ for 19 dof) using the quadratic function (shown in left lower panel of Figure 11). The new curve is somewhat flatter than the old one and leads to an updated quadratic ephemeris given by:

$$
\begin{array}{r}
T_{\text {elc }}=2445615.30927(41)+ \\
+0.232109006(67) N \\
-0.96(18) \times 10^{-11} N^{2}
\end{array}
$$

Where $\mathrm{N}$ is the cycle number and linear ephemeris, and errors are at $90 \%$ confidence on the last 2 digits. For comparison, the dotted line shows the quadratic fit obtained for the old X-ray datasets by Parmar et al. (2000). The new X-ray eclipse times derived from the HETG observations are given in table 2 with uncertainties given at $90 \%$ confidence.

\subsection{Phase Resolved Line Properties}

Observed spectral features are highly phase-dependent. The new observations cover almost four times the number of binary orbits than what Cottam et al. (2001) had available, which allow us to investigate line variations with orbital phase in much more detail. The lower right panel in Figure 1 shows the phase separation for our stronger line detections. We center the first phase bin at eclipse minimum. To study the H-like and Helike lines, except the narrow phase bin $(\sim 0.08)$ during eclipse, we choose a phase bin width of 0.16 . This secures enough counts for the line features in each bin, and the Ne X, Ne IX, Mg XII, Mg XI, O VIII, and O VII emission lines are clearly detected with this choice. We divide the orbit into 40 even, but overlapping, phase segments. The Fe XXVI and Fe K $\alpha$ have very weak signal with this choice of (correlated) phase bin, consequently, we additionally chose a set of five, non-overlapping phase bins as marked in upper right panel of Figure 11. They are denoted as upper(1), decreasing(2), bottom(3), eclipsing(4), and rising(5) respectively.

\subsubsection{Fe lines}

The crude five phase binning of the Fe XXVI and $\mathrm{Fe} \mathrm{K} \alpha$ lines smears out most orbital information. Detections are shown in Table 5, and no line shifts are found. However, their flux trends follow the shape of the light curves, reaching a minimum during eclipse and a maximum at the light curve's maximum. For all five phases, the centroid of the $\mathrm{Fe} \mathrm{K}$ line is consistent with $1.93 \AA$ which covers wavelengths of Fe I to Fe XX from the cold, nearneutral medium, similar to the finding by Cottam et al. (2001). However, we find a lower limit to the line broadening of about $370 \mathrm{~km} \mathrm{~s}^{-1}$.

\subsubsection{Bright Line Dynamics}

The preliminary analysis in Cottam et al. (2001) only had two binary orbits at hand, which provided four phase bins and tentative suggestions with respect to an orbital phase dependence of bright line centroids. Incorporating the seven orbits in the new observations allows for a much more detailed analysis of the line phase dependence. These line properties include line centroids, line widths, and line fluxes. The results are shown in Figures 5 (for Ly $\alpha$ lines) and 6 (for intercombination lines) and Tables 3 and 4 . These are the brightest lines in the X-ray spectrum. There are other weaker lines present in the spectrum; however, we perform the phase dependent analysis only for the bright lines. 
All of the lines show significant wavelength shifts with orbital phase. Most lines follow a very similar shift pattern. At phase 0 the lines appear highly red-shifted and exhibit values between $400-500 \mathrm{~km} \mathrm{~s}^{-1}$. These values decline to zero near about phase 0.25 and turn into increasing blue-shifts of up to $400-500 \mathrm{~km} \mathrm{~s}^{-1}$ near phase 0.5 where in most cases the shifts reverse again. The most prominent lines showing this pattern are Ne IX, Mg XI, $\mathrm{Mg}$ XII, and Si XIV. In Ne Xthe shift reversal happens later at phase 0.8 , at $\mathrm{O}$ VIIand $\mathrm{O}$ VIII somewhat earlier than mid-phase. The wave pattern appears most clearly in Ne IX, Mg XII, and Si XIV, which are also the brightest in the sample. Uncertainties vary between 100 and 250 $\mathrm{km} \mathrm{s}^{-1}$ depending on the phase and the detected flux. This pattern is very consistent with a spatially confined emission region moving with orbital phase. We illustrate this with a cartoon structure of the system and the phase light curve highlighting the phase bin location as well as the line spectrum for the case of Ne IX in Figure 7 Shown phases include $0,0.1,0.42,0.68,0.83$, and 0.92 .

The second part in Figure [6] also shows the velocity width of the lines. Even though there appears to be some subtle variations with phase, in most cases the widths appear rather stable over the entire orbit. The velocity widths for all lines appear very similar near $400 \mathrm{~km} \mathrm{~s}^{-1}$ with uncertainties of around 150 $\mathrm{km} \mathrm{s}^{-1}$ per phase bin, again depending on detected flux. The very similar widths are consistent with a radius of $(1.14 \pm 0.7) \times 10^{11} \mathrm{~cm}$.

Table 7 and Figure 4 list the results of our detections for He-like lines of O VII, Ne IX, and Mg XI. The intercombination line flux is very bright and appears broad, while the resonance line is weak, and we do not detect the forbidden line component. The resulting $G$ ratios, defined as $(\mathrm{i}+\mathrm{f}) / \mathrm{r}$, are all consistent with a pure recombination plasma. The $\mathrm{R}$ ratios, defined as $\mathrm{f} / \mathrm{i}$, are all very small indicating either high levels of photo-excitation or the presence of a high density plasma. To remove the orbital smear we compute the R and G values for the five phase bin case, which are shown in Figure 3 and Table 6 . In orbital phases 2 and 4 we do not have enough statistics to compute values, however for phases 1,3 , and 5 we compute values and value limits which are consistent with each other.

\subsection{The Photo-ionized X-ray Spectrum}

We fit spectra for the five phase bins described in Sect. 3.2 and find that it can be fit well at most phases using a cut-off power law plus a soft black body component. The norm variations in both components follow the flux change in the light curve. The power law index is very flat with values in the range $\approx-0.4--0.2$. This flat power law reflects the fact that we likely observe indirect emission affected by a central hot corona as already found in RXTE and ASCA data (Heinz \& Nowak 2001). The blackbody component has an average temperature of $181 \pm 11 \mathrm{eV}$ with slight variations, which possibly reflects some correlation with the photo-electric absorption function in the fit. We detect some significant absorption using the updated Tbnew function in Xspec of $(1.6 \pm 0.3) \times 10^{21} \mathrm{~cm}^{-2}$ throughout all phases, which is near the upper limit presented by Heinz \& Nowak (2001). The highest blackbody flux in the light curve amounts to $3 \times 10^{34} \mathrm{erg} \mathrm{s}^{-1}$, which we estimate is only about $2 \%$ of the total flux (see below) and provides an emission radius of about $20 \mathrm{~km}$. Figure 8 (left) shows the continuum fit to phase 1 with blackbody, power-law, and gaussian line components.

Alternatively it has also been suggested by Heinz \& Nowak (2001) and others that the spectra should be fit using the power law with additional partial covering absorption. We have simultaneously fit phases 1-5 with such a function wherein we forced the power law slope to be the same for all phases (the fit yields $\Gamma=0.656 \pm 0.002$, with $90 \%$ confidence error bars), but let the power law normalization, line parameters, partial covering fraction, and covering column be free parameters for each phase. Figure 8 (right) shows the results of this fit for the phase 1 spectra, with the photo-ionized lines represented by gaussian functions. We find that this model simultaneously fits all 5 spectra very well $\left(\chi^{2}=2266.6 / 2176 \mathrm{DoF}\right)$. The direct and covered power law represent a normalized flux that ranges from $0.57-1$. That is, at least $43 \%$ of the total power law flux is completely blocked in the faintest phase, i.e., phase 4. (In all likelihood, a larger fraction of the ADC flux is completely blocked, as phase 1 is unlikely to be completely uncovered by the disk rim.) The partial covering fraction varies between $49-52 \%$, while the partial covering column varies between $5.4-6.3 \times 10^{22} \mathrm{~cm}^{-2}$. In this case the ISM absorption remains very low at $(2.3 \pm 0.3) \times 10^{20} \mathrm{~cm}^{-2}$.

The line emitting region is best viewed during phases 1 and 5 , with the least dynamical smear during phase 5 . During phase 1 the line emitting region is more directly viewed, but here the lines switch from red-shift to blueshift and the region is likely already partially obscured by the central corona. Figure 3 thus shows the Ne IX line strongest and most narrow. We select phase bin 5 for the more detailed spectral modeling. The main goal of this analysis is to get an estimate for the ionization parameter of the emitting region. Photo-ionization properties are generally described by the parameter $\xi=L /\left(n * r^{2}\right)$, where $\mathrm{L}$ is the source $\mathrm{X}$-ray luminosity, $\mathrm{n}$ is the electron density, and $\mathrm{r}$ is the distance to the illuminating X-ray source (Kallman \& McCray 1982). From Figure 8 we already see that the $\mathrm{O}$ VII and $\mathrm{O}$ VIII line strengths are similar, which is difficult to achieve with a single ionization parameter. We thus expect a range of parameters.

We want to keep this analysis as simple as possible and use the new embedded Xstar function photemis in Xspec. Fitting the lines with a single ionization parameter, we obtain $\log \xi=2.6[\mathrm{erg} \mathrm{cm} / \mathrm{s}]$, which fits the $\mathrm{H}$-like ion lines very well but not the He-like ion species. However, even though a second ionization parameter of $\log \xi=1.9[\mathrm{erg} \mathrm{cm} / \mathrm{s}]$ would fit these lines, the fit itself is not acceptable as it would produce significant radiative recombination continua (RRC) for O VIII, Ne IX and Ne X, which we do not observe. A viable solution to make up for this deficiency is to assume a shallow tail of lower ionization parameters (i.e. below $\log \xi=2.6[\mathrm{erg} \mathrm{cm} / \mathrm{s}]$ ), allowing for high enough plasma temperatures to smear out the RRCs but produce enough line flux to fit the Helike lines. The range of this tail depends on abundance choices and here we cannot find unique solutions.

\section{DISCUSSION}


$4 \mathrm{U} 1822-371$ is known as one of the prototypical ADC systems and shows a strong orbital dependence of its Xray properties. Our coverage of many orbital periods allows us to study these dependencies in detail. The updated eclipse times from the three Chandra observations are not inconsistent with the trend provided by previously published X-ray eclipse times (Parmar et al. 2000). But even though our result does follow the quadratic function implied by these previous observations, it does suggest a flatter trend. This is still consistent with a previous result, which provided the last updated improved ephemeris of 4U 1822-371 derived from UV/optical light curves (Bavless et al. 2009). But in their analysis, the quadratic term in the optical is also consistent with the one in X-ray by Parmar et al. (2000) within the measurement errors, and the eclipse time in the optical lags behind by about 100 seconds. More recently, Burderi et al. (2010) revisited X-ray observations over the last 30 yrs confirming the quadratic function by Parmar et al. (2000), but did not include recent optical and UV data. In their analysis the eclipse times from our Chandra observations showed much larger delays which appear closer to the quadratic function by Parmar et al. (2000), however the delays also remained significantly below the function. One possible explanation for the discrepancy with our result is that they opted not to fold the light curves, which we think is warranted given the multiple eclipses within these data, and the fact that we see intermittent dips in the spectra, some occuring quite close to the main eclipses. In any case, the trend solidifies, that the orbital period change in this system is larger than expected from simple magnetic braking and losses due to gravitational radiation.

The line variations with orbital phase can probe geometrical properties such as accretion disk size, ADC size, and also a phase-resolved ionization balance of the line emitting region. While most of the lower $\mathrm{Z}$ ions show significant dynamical properties along the binary orbit with respect to all of their line properties, the iron lines only show flux changes consistent with the changes observed in the light curves. The Fe $\mathrm{K} \alpha$ fluorescence line is visible at all phases. Its flux is strongest opposite of the eclipse, when the disk is fully exposed to the observer. During eclipse the fact that the line flux is still about $30 \%$ of its maximum indicates that the line emitting region extends significantly beyond the size of the companion. We also do not believe that the illuminated surface of the stellar companion is a major source of Fe fluorescence, because at an edge-on view and opposite to eclipse the disk would likely block the flux and we would expect maxima during the decreasing and bottom phases (Table 5). Similarly, using fluorescence probabilities calculated by Bai (1979) we also expect maxima at these phases and not at the opposite (upper) phase. Instead we argue, consistent with the suggestions by Cottam et al. (2001), that the fluorescence materials come from an extended region above the disk. In fact, as Torrejon, et al. (2010) showed in a large survey of Fe K fluorescence in X-ray binaries including $4 \mathrm{U} 1822-371$ that the emissions come from a large, spherically distributed volume around the accretion disk. The width of Fe K $\alpha$ fluorescence line are also better constrained than the previous detection by Cottam et al. (2001) and we determine a lower limit of the emission radius of $R \geq 1 \times 10^{9.5} \mathrm{~cm}$. We do not know how well this fits into the previous conclusion by Bayless et al. (2009) with respect to the existence of a layer of cooler material at the base of the wind seen in the UV, but the $\mathrm{X}$-ray data do not exclude this possibility.

We also detect weak line emissions from hot Fe XXVI ions. As is observed for the cool Fe $\mathrm{K} \alpha$ fluorescence, the Fe XxVI, Lyman $\alpha$ line does not show any line shifts and broadening with respect to orbital phases. Its flux roughly follows that of the light curve and is below detection during eclipse. This indicates that the line originates from a central region within the disk radius, likely from parts of the central corona but not the disk itself. Its emission volume is then limited by the size of the companion during eclipse. Its Roche-lobe filling radius is about $0.54 R_{\odot} \quad$ (Bayless et al. 2009) and thus the emission size is less than $3.5 \times 10^{10} \mathrm{~cm}$ and thus about 0.65 $\mathrm{r}_{\text {disk }}$ assuming an orbital separation of $\mathrm{a}=1.33 \times 10^{11} \mathrm{~cm}$ and a disk radius 0.4 a (values taken from Bayless et al. 2009).

Photo-ionized X-ray line emission in 4U 1822-371 has been observed previously (Cottam et al. 2001) and phase-resolved spectra suggested that the emissions from line recombination originate in an X-ray illuminated bulge located at or near the predicted point of impact of the accretion stream from the companion and the disk. Our observations over several binary orbits not only confirm this prediction, but allow us to study this phenomenon in detail. The lower $\mathrm{Z}$ line emissions clearly show red- and blue-shifted motion with orbital phase which can only be interpreted by motion of a localized line emitting area at the hot spot. The maximum absolute velocities from the line shifts of the most prominent lines (except Fe XXVI, see above), i.e., O VII, O VIII, Ne IX, Ne X, Mg XI, Mg XII, Si XIV, yield a velocity of $500 \mathrm{~km} \mathrm{~s}^{-1}$, which is consistent with the orbital speed projection expected from the orbital parameters of $4 \mathrm{U}$ $1822-371$ at the inclination of $83^{\circ}$. Using 40 overlapping phase bins we observe the lines moving at all times and variations in the line widths appear very small, which restricts the size of the emitting area to $<10^{6} \mathrm{~cm}$. In fact, the very similar width in all lines places the emitting region at a consistent distance of $1.1 \times 10^{11} \mathrm{~cm}$ to the source, which is about twice the actual outer disk radius and more close to the separation of the two stars. However, even though the uncertainties in the width include the outer disk radius we predict that there is local turbulence involved in the width.

There are a few more interesting implications from the broadband analysis of the X-ray spectrum. The fact that we fit a soft blackbody spectrum to the soft part of the X-ray spectrum is consistent with one solution obtained from the ASCA analysis (Heinz \& Nowak 2001); however, the blackbody temperature we measure is very much lower. The properties we determine provide an emission radius close to $20 \mathrm{~km}$, which appears like the emission from the neutron star itself. However, the line emission properties imply a radically different picture. As Heinz \& Nowak (2001) already suspected in their analysis of the $A S C A$ data, we likely do not observe the X-ray source directly and the true source flux should be considerably higher. The ionization parameter of the line emitting region is directly related to the illuminat- 
Table 1

Observations for $4 \mathrm{U} 1822-371$

\begin{tabular}{l|cccl}
\hline \hline obsID & MJD interval & Obs. Start & Exp.(ks) & PI \\
\hline 671 & $51779.681-51780.163$ & $2000-08-23 ~ 16: 20: 37$ UT & 39.95 & Kahn, M. \\
9076 & $54606.949-54607.708$ & $2008-05-20 ~ 22: 46: 21$ UT & 63.68 & Canizares, C. \\
9858 & $54609.551-54610.524$ & $2008-05-23$ 13:14:05 UT & 82.19 & Canizares, C. \\
& & & & \\
\hline
\end{tabular}

ing source flux (see Sect. 3.3). At values between $\log \xi$ $=1.9-2.6$, a disk radius of $5 \times 10^{10} \mathrm{~cm}$, and a plasma density between $10^{12}$ and $10^{14} \mathrm{~cm}^{-3}$, for the thin illuminated layer the illuminating luminosity has to be much larger than $10^{37} \mathrm{erg} \mathrm{s}^{-1}$. This also has a consequence for the blackbody emitting radius, which once adjusted to the higher source luminosity is more of the order of $>100 \mathrm{~km}$. This makes the solution with the blackbody component highly unattractive as it removes the possibility that we see the neutron star, but it is also rather weak to account for inner disk emissions. In this respect we argue for the solution with the partial covering component, i.e., the X-ray source is a power law in nature, likely scattered into our line of sight over the spatially very extended corona, and due to the edge-on view we observe some of this emission both blocked and heavily absorbed by the disk rim.

\section{CONCLUSIONS}

The orbital phase-resolved analysis of the X-ray photoionized region in $4 \mathrm{U} 1822-37$ obtained the following results:

- The orbital X-ray ephemeris update suggests a flatter trend with respect to the previously proposed quadratic change function.

- Fe xxvi line emission arises from a central corona of size $<3.5 \times 10^{10} \mathrm{~cm}$.

- Lower Z line emissions show orbital shifts consistent with a small local region at the rim of the disk, exhibiting orbital blue- and red-shifts of the order of $500 \mathrm{~km} \mathrm{~s}^{-1}$.

- The line emitting region has to be the inner illuminated side of a bulge at the hot spot because it becomes entirely eclipsed when the outer side of the spot faces the observer.

- The latter fact also implies that the illuminated layer on top of the disk is thin, at least thin enough not to exceed the outer disk rim.
- Line widths appear larger than the outer disk radius and likely involve local turbulence.

- The line emitting region exhibits high ionization parameters, while missing RRCs constrain values to be above $\log \xi>2$. This strongly suggests that we do not directly observe the X-ray source.

- The luminosity implied by the range of ionization parameters favors an emission model which consists of a cut-off power law plus a $50 \%$ partial covering absorption.

We thank Mike Noble, John Houck for their help with ISIS. We gratefully acknowledge the financial support of Chandra X-Ray Observatory archive grant AR0-11005X.

\section{REFERENCES}

Bai, T., 1979, Solar Physics, 62, 113

Bayless, A.J., Robinson, E.L., Hvnes, R.I., Ashcraft, T.A., \& Cornell, M.E., 2009, astro-ph/09114492

Burderi, L.. DiSalvo, T., Kiggio, A., et al., 2010, astro-ph/1006328,

Canizares, C.R., et al. 2000, ApJ, 539, L41

Casares, J., Steeghs, D., Hynes, R.I., et al., 2003, ApJ, 590, 1041

Cottam, J., Sako, M., Kahn, S.M., \& Paerels, F., 2001, ApJ, 557, L101

Cowley, A.P., Schmidtke, P.C., Hutchings, J.B., Crampton, D., 2003, AJ, 125, 2163

Harlaftis, E.T., Charles, P.A., \& Horne, K., 1997, MNRAS, 285, 673

Heinz, S., \& Nowak, M.A., 2001, MNRAS, 320, 249

Hellier, C. \& Mason, K.O., 1989, MNRAS, 239, 715

Houck, J.C. \& Denicola, L.A. 2000, in ASP Conf. Ser. 216,

Astronomical Data Analysis Software and Systems IX, ed. N. Manset, C. Veillet, \& D. Crabtree (San Francisco, CA: ASP), 591

Jonker, P.G., \& van der Klis, M., 2001, ApJ, 553, L43

Kallman, T. R., \& McCray, R., 1982, ApJS, 50, 263

Mason, K.O., \& Cordova, F.A., 1982, ApJ, 255, 603

Parmar, A.N., Oosterbroek, T., Del Sordo, S., et al., 2000, A\&A, 356,175

Torrejon, J.M., Schulz, N.S., Nowak, M., Kallman, T.K., 2010, A\&A, 715,947

White, N.E., Becket, R.H., Boldt, E.A., et al., 1981, ApJ, 247, 994

White, N.E., \& Holt, S.S., 1982, ApJ, 257, 318

White, N.E., Kallman, T.R., \& Angelini, L., Proceedings of an International Symposium on X-ray Astronomy ASCA Third Anniversary, 11-14 March, 1996, Waseda University, Tokyo. Edited by F. Makino and K. Mitsuda (1997), p411 
Table 2

New X-ray eclipse times for 4 U1822-371 with uncertainties given at $90 \%$ confidence.

\begin{tabular}{l|ccc}
\hline \hline obsID & $\mathrm{JD}_{\odot}$ & Uncertainty & Cycle \\
\hline 671 & 2451780.36424 & 0.00079 & 26561 \\
9076 & 2454607.69102 & 0.00045 & 38742 \\
9858 & 2454610.24377 & 0.00037 & 38753 \\
& & & \\
\hline
\end{tabular}


Table 3

Ly $\alpha$ lines along the phases for $4 \mathrm{U} 1822-371^{\mathrm{a}}$

\begin{tabular}{|c|c|c|c|c|c|c|c|c|c|c|c|}
\hline \multirow[t]{2}{*}{ phase } & \multicolumn{3}{|c|}{ SiXIV Ly $\alpha$} & \multicolumn{3}{|c|}{$\operatorname{MgXII} \operatorname{Ly} \alpha$} & \multicolumn{3}{|c|}{$\operatorname{NeX} \operatorname{Ly} \alpha$} & \multicolumn{2}{|r|}{ OVIII Ly $\alpha$} \\
\hline & $\lambda$ & Flux & $\sigma$ & $\lambda$ & Flux & $\sigma$ & $\lambda$ & Flux & $\sigma$ & $\lambda$ & Flux \\
\hline 10.0 & $.185_{-0.005}^{+0.005}$ & $2.6_{-1}^{+1}$. & 1.0 & $8.435_{-0.013}^{+0.016}$ & $2.7_{-1.8}^{+2.3}$ & $14.0_{-6.3}^{+9.0 \mathrm{~b}}$ & - & - & - & $18.997_{-0.037}^{+0.037}$ & $7.5_{-66}^{+1.3}$ \\
\hline 20.025 & & $2.4_{-1.2}^{+1.5}$ & $<15.0$ & $441_{-0.010}^{+0.005}$ & $1.9_{-1.0}^{+1.6}$ & $<13.2$ & $12.149_{-0.005}^{+0.010}$ & $2.9_{-1.4}^{+2.6}$ & $<19$ & $18.994_{-0.008}^{+0.007}$ & $9.6_{-5.2}^{+6.9}$ \\
\hline 30.05 & $\begin{array}{l}005 \\
005\end{array}$ & $2.7_{-1.0}^{+1.1}$ & 1.0 & $8.436_{-0.010}^{+0.010}$ & $2.7_{-1.4}^{+1.6}$ & $13.2_{-9.3}^{+11.2}$ & $12.149_{-0.010}^{+0.010}$ & $5.2_{-2.6}^{+2.6}$ & $16.6_{-6.0}^{+6.7 \mathrm{~b}}$ & $18.995_{-0.008}^{+0.008}$ & $\begin{array}{c}0.5 .2 \\
12.8_{-7.3}^{+9.2}\end{array}$ \\
\hline 40.075 & $\begin{array}{l}004 \\
003\end{array}$ & $4.0_{-1.4}^{+1.6}$ & $4.8_{-3}^{+3}$ & $8.433_{-0.009}^{+0.009}$ & $2.9_{-1.4}^{+1.7}$ & $13.3_{-9.9}^{+11.8}$ & $12.144_{-0.009}^{+0.009}$ & $7.1_{-2.5}^{+2.8}$ & $19.1_{-8.6}^{+9.8}$ & $18.993_{-0.009}^{+0.007}$ & $6.8_{-4.3}^{+5.3}$ \\
\hline 50.1 & $\begin{array}{l}.003 \\
.003\end{array}$ & $4.1_{-1.4}^{+1.6}$ & $\begin{array}{r}<9.4 \\
\end{array}$ & $8.430_{-0.007}^{+0.007}$ & $3.2_{-1.4}^{+1.6}$ & $10.8_{-8.6}^{+8.4}$ & $12.145_{-0.008}^{+0.009}$ & $8.6_{-2.7}^{+3.1}$ & $20.1_{-}^{+}$ & $18.993_{-0.009}^{+0.011}$ & $12.3_{-6.1}^{+8.8}$ \\
\hline 60.125 & $92_{-0.003}^{+0.003}$ & $3.8_{-1.3}^{+1.5}$ & $<7.8$ & $8.432_{-0.005}^{+0.006}$ & $3.0_{-1.3}^{+1.5}$ & $7.9_{-4.3}^{+4.56}$ & $12.145_{-0.007}^{+0.007}$ & $7.5_{-2.6}^{+2.8}$ & $15.4_{-7.4}^{+7.0}$ & $18.999_{-0.008}^{+0.009}$ & $16.4_{-7.1}^{+9.1}$ \\
\hline 70.15 & $\begin{array}{l}.004 \\
0.004\end{array}$ & $4.1_{-1.5}^{+1.8}$ & $4.9_{-3}^{+3}$ & $8.430_{-0.006}^{+0.006}$ & $3.7_{-1.6}^{+1.9}$ & $8.9_{-6.3}^{+5.9 b}$ & $12.144_{-0.007}^{+0.008}$ & $7.3_{-2.7}^{+2.9}$ & $15.3_{-10.5}^{+8.6}$ & $18.992_{-0.010}^{+0.011}$ & $22.1_{-8.8}^{+10.5}$ \\
\hline 80.175 & $\begin{array}{l}0.005 \\
.005 \\
.005\end{array}$ & $4.5_{-1.8}^{+\frac{1}{2} .0}$ & $8.6_{-6.2}^{+6.2}$ & $8.430_{-0.004}^{+0.006}$ & $3.1_{-1.1}^{+1.0}$ & $\begin{array}{l}-6.3 \\
<8.7\end{array}$ & $12.146_{-0.007}^{+0.007}$ & $8.0_{-2.5}^{+2.8}$ & $16.2_{-5.0}^{+6.8}$ & $18.994_{-0.013}^{+0.013}$ & $26.0_{-9.9}^{+12.1}$ \\
\hline 90.2 & $\begin{array}{l}006 \\
006\end{array}$ & $4.2_{-1.8}^{+2.2}$ & $9.3_{-6.2}^{+9.2}$ & $8.427_{-0.006}^{+0.005}$ & $4.1_{-1.8}^{+1.8}$ & $9.5_{-6.4}^{+4.2 b}$ & $12.143_{-0.006}^{+0.006}$ & $9.2_{-2.6}^{+2.8}$ & $16.1_{-4.8}^{+6.0}$ & $18.983_{-0.015}^{+0.020}$ & $32.4_{-11.4}^{+13.9}$ \\
\hline 100. & $\begin{array}{l}.005 \\
.005\end{array}$ & $5.3_{-1.8}^{+2.0}$ & $9.7_{-4}^{+5}$ & $8.428_{-0.004}^{+0.004}$ & $3.1_{-1.2}^{+1.5}$ & $\begin{array}{l}-0.4 \\
<8.9\end{array}$ & $12.140_{-0.006}^{+0.006}$ & $9.7_{-2.6}^{+2.9}$ & $16.3_{-4.9}^{+5.8}$ & $18.983_{-0.015}^{+0.015}$ & $22.9_{-9.9}^{+11.4}$ \\
\hline 110. & $\begin{array}{l}0.004 \\
0.004\end{array}$ & $5.3_{-1.8}^{+2.0}$ & $8.9_{-3.7}^{+4.8}$ & $8.428_{-0.008}^{+0.007}$ & $3.8_{-1.6}^{+1.8}$ & $12.8_{-7.7}^{+8.4}$ & $12.146_{-0.008}^{+0.008}$ & $7.8_{-2.6}^{+2.8}$ & $17.1_{-5.7}^{+7.2}$ & $18.980_{-0.017}^{+0.019}$ & $21.4_{-9.3}^{+11.2}$ \\
\hline 120.275 & $\begin{array}{l}.006 \\
.005\end{array}$ & $4.5_{-1.8}^{+\frac{1}{2} .0}$ & $9.1_{-4.9}^{+6.1}$ & $8.427_{-0.008}^{+0.007}$ & $4.7_{-1.7}^{+1.6}$ & $15.3_{-6.5}^{+7.7}$ & $12.137_{-0.009}^{+0.008}$ & $7.9_{-2.7}^{+3.0}$ & $19.5_{-6.2}^{+7.7}$ & $18.974_{-0.022}^{+0.023}$ & $24.5_{-10.8}^{+12.3}$ \\
\hline 130.3 & .006 & $4.7_{-1.8}^{+2.2}$ & $9.9_{-7.4}^{+7.3}$ & $8.428_{-0.007}^{+0.007}$ & $4.3_{-1.7}^{+1.9}$ & $13.8_{-7.0}^{+8.3}$ & $12.136_{-0.008}^{+0.009}$ & $8.3_{-2.7}^{+3.0}$ & $18.6_{-6.6}^{+8.3}$ & $18.957_{-0.013}^{+0.013}$ & $18.4_{-7.8}^{+8.9}$ \\
\hline 40. & $\begin{array}{l}.008 \\
.0007\end{array}$ & $3.4_{-1.9}^{+1.8}$ & $8.9_{-4.4}^{+4.3 \mathrm{~b}}$ & $8.426_{-0.010}^{+0.008}$ & $4.1_{-1.8}^{+2.3}$ & $15.3_{-8.6}^{+1.0}$ & $12.136_{-0.008}^{+0.008}$ & $9.4_{-2.8}^{+3.2}$ & $19.1_{-6.5}^{+8.6}$ & $18.945_{-0.010}^{+0.011}$ & $14.7_{-7.6}^{+9.4}$ \\
\hline 150.3 & .008 & $3.6_{-2.3}^{+2.3}$ & $10.1_{-9.4}^{+9.4}$ & $8.425_{-0.008}^{+0.008}$ & $3.9_{-1.7}^{+2.1}$ & $13.8_{-8.2}^{+11.5}$ & $12.138_{-0.009}^{+0.008}$ & $8.3_{-2.9}^{+3.2}$ & $20.2_{-7.3}^{+8.5}$ & $18.949_{-0.010}^{+0.010}$ & $11.8_{-6.2}^{+8.6}$ \\
\hline 160.375 & $\begin{array}{l}0.010 \\
0.015\end{array}$ & $3.0_{-2.0}^{+1.1}$ & $<58.2$ & $8.421_{-0.012}^{+0.010}$ & $4.5_{-2.0}^{+2.7}$ & $17.7_{-8.4}^{+17.2}$ & $12.136_{-0.009}^{+0.010}$ & $8.3_{-2.9}^{+3.3}$ & $18.9_{-7.3}^{+9.4}$ & $18.990_{-0.044}^{+0.028}$ & $11.5_{-8.9}^{+14.4}$ \\
\hline 170.4 & $\begin{array}{l}0.015 \\
0.005\end{array}$ & $2.5_{-1.3}^{+1.3}$ & 1.0 & $8.423_{-0.011}^{+0.008}$ & $3.8_{-1.8}^{+2.3}$ & $14.7_{-7.9}^{+14.1}$ & $12.137_{-0.009}^{+0.010}$ & $8.7_{-3.1}^{+3.4}$ & $20.0_{-8.1}^{+9.8}$ & $18.970_{-0.026}^{+0.019}$ & $24.5_{-11.0}^{+13.8}$ \\
\hline 180. & $\begin{array}{l}.003 \\
.004\end{array}$ & $3.0_{-1.3}^{+1.3}$ & 1.0 & $8.420_{-0.009}^{+0.011}$ & $2.7_{-1.4}^{+1.8}$ & $11.5_{-5.7}^{+13.1}$ & $12.139_{-0.009}^{+0.007}$ & $7.8_{-3.2}^{+3.4}$ & $15.2_{-9.9}^{+12.1}$ & $18.975_{-0.024}^{+0.020}$ & $19.6_{-9.6}^{+11.5}$ \\
\hline 190.4 & $6.175_{-0.004}^{+0.005}$ & $3.0_{-1.3}^{+1.3}$ & 1.0 & $8.414_{-0.011}^{+0.011}$ & $2.8_{-1.5}^{+1.8}$ & $10.6_{-4.5}^{+6.0 \mathrm{~b}}$ & $12.141_{-0.009}^{+0.008}$ & $7.8_{-2.9}^{+3.3}$ & $16.2_{-9.9}^{+9.7}$ & $18.981_{-0.017}^{+0.016}$ & $20.8_{-9.2}^{+11.0}$ \\
\hline 200. & $\begin{array}{l}.011 \\
.007\end{array}$ & $3.7_{-2.1}^{+77.4}$ & $9.3_{-5.5}^{+7.2 \mathrm{~b}}$ & $8.404_{-0.004}^{+0.010}$ & $2.2_{-1.0}^{+1.1}$ & $\begin{array}{l}-4.5 \\
<8.6\end{array}$ & $12.138_{-0.010}^{+0.009}$ & $7.1_{-2.8}^{+3.2}$ & $17.9_{-8.9}^{+10.9}$ & $18.987_{-0.017}^{+0.017}$ & $15.3_{-8.0}^{+9.2}$ \\
\hline 210.5 & $\begin{array}{l}.003 \\
.004\end{array}$ & $2.8_{-1.3}^{+1.3}$ & 1.0 & $8.404_{-0.004}^{+0.004}$ & $1.8_{-1.0}^{+l .0}$ & 1.0 & $12.135_{-0}^{+0}$ & $6.8_{-2.9}^{+3.3}$ & $20.3_{-10.0}^{+12.2}$ & $18.986_{-0.017}^{+0.017}$ & $16.2_{-8.0}^{+9.8}$ \\
\hline 220. & & $3.1_{-1.8}^{+2.0}$ & $8.2_{-4.1}^{+4.9 \mathrm{~b}}$ & $8.401_{-0.005}^{+0.004}$ & $1.5_{-0.9}^{+l .0}$ & 1.0 & $12.131_{-0.018}^{+0.019}$ & $5.4_{-2.8}^{+3.3}$ & $22.7_{-11.3}^{+17.8}$ & $18.996_{-0.028}^{+0.013}$ & $11.7_{-6.8}^{+10.5}$ \\
\hline 230.55 & $\begin{array}{l}010 \\
009\end{array}$ & $3.8_{-2.0}^{+2.8}$ & $13.0_{-4.3}^{+5.4 \mathrm{~b}}$ & $8.403_{-0.005}^{+0.003}$ & $1.6_{-0.9}^{+0.9}$ & 1.0 & $12.142_{-0.020}^{+0.018}$ & $4.0_{-2.4}^{+3.2}$ & $16.8_{-12.5}^{+23.6}$ & $18.992_{-0.020}^{+0.028}$ & $20.7_{-9.6}^{+11.8}$ \\
\hline 240.575 & $6.186_{-0 .}^{+0 .}$ & $3.7_{-2.1}^{+2.6}$ & $14.6_{-10.2}^{+13.9}$ & $8.405_{-0.007}^{+0.005}$ & $1.6_{-0.9}^{+0.0}$ & 1.0 & $12.139_{-0.019}^{+0.020}$ & $4.3_{-2.7}^{+3.4}$ & $19.7_{-10.3}^{+24.0}$ & $18.990_{-0.021}^{+0.024}$ & $21.2_{-10.3}^{+11.6}$ \\
\hline 250.6 & - & - & - & - & -0 & - & $12.127_{-0.021}^{+0.019}$ & $1.5_{-1.3}^{+1.8}$ & $16.6_{-11.4}^{+12.0}$ & $18.975_{-0.022}^{+0.022}$ & $16.6_{-8.4}^{+9.8}$ \\
\hline 260.625 & $6.182_{-0.007}^{+0.006}$ & $1.6_{-1.1}^{+1.1}$ & 1.0 & - & - & - & $12.129_{-0.012}^{+0.013}$ & $3.6_{-2.3}^{+2.3}$ & $13.5_{-10.0}^{+6.5 .4}$ & $18.982_{-0.026}^{+0.038}$ & $14.1_{-7.4}^{+11.4}$ \\
\hline 270 & - & - & - & - & - & - & $12.133_{-0.016}^{+0.014}$ & $3.8_{-2.4}^{+2.4}$ & $16.6_{-8.3}^{+12.4}$ & $18.976_{-0.021}^{+0.022}$ & $15.6_{-8.0}^{+9.8}$ \\
\hline 280.6 & $92_{-0.010}^{+0.009}$ & $3.6_{-1.8}^{+2.2}$ & $13.9_{-6.9}^{+10.3}$ & - & - & - & $12.127_{-0.007}^{+0.019}$ & $3.1_{-1.7}^{+3.3}$ & $<21.6$ & $18.985_{-0.027}^{+0.027}$ & $13.0_{-7.7}^{+9.4}$ \\
\hline 290.7 & $\begin{array}{l}007 \\
008\end{array}$ & $3.9_{-1.7}^{+2.0}$ & $12.2_{-5.9}^{+8.1}$ & - & - & - & $12.125_{-0.006}^{+0.015}$ & $3.6_{-1.8}^{+2.8}$ & $<15.7$ & - & - \\
\hline 300. & 07 & $3.0_{-1.5}^{+1.8}$ & $\begin{array}{l}9.0_{-6.6}^{+8.8} \\
{ }^{+8.9}\end{array}$ & - & - & - & $12.124_{-0.005}^{+0.008}$ & $3.5_{-1.6}^{+2.0}$ & $<10.9$ & - & - \\
\hline & $\begin{array}{l}0 \\
110 \\
10\end{array}$ & $2.7_{-1.7}^{+1.9}$ & 9. & - & - & - & $12.122_{-0.006}^{+0.010}$ & $2.9_{-1.5}^{+1.6}$ & $<9.0$ & - & \\
\hline & & $2.8_{-1.6}^{+1.1}$ & $7.8_{-5}^{+4}$ & — & - & - & - & -1.0 & - & $19.004_{-0.009}^{+0.010}$ & $3.9_{-3.1}^{+4.9}$ \\
\hline 330.8 & $\begin{array}{l}008 \\
008 \\
008\end{array}$ & $2.8_{-1.7}^{+1.6}$ & $8.6_{-6.4}^{+5.8 \mathrm{~b}}$ & - & - & - & 一 & - & - & $19.003_{-0.008}^{+0.012}$ & $4.4_{-3.3}^{+5.1}$ \\
\hline & $\begin{array}{l}010 \\
011\end{array}$ & $2.3_{-1.5}^{+2.1}$ & $9.7_{-7.2}^{+6.4} \mathrm{~b}$ & $8.436_{-0.005}^{+0.005}$ & $1.3_{-0.8}^{+0.9}$ & 1.0 & - & & - & $19.005_{-0.009}^{+0.010}$ & $4.9_{-3.6}^{+5.3}$ \\
\hline 350. & $.190_{-0.011}^{+0.011}$ & $3.0_{-1.9}^{+\frac{1}{2} .3}$ & $13.1_{-6.5}^{+7.2 b}$ & $8.436_{-0}^{+0}$ & $1.6_{-0.8}^{+0.8}$ & 1.0 & $12.152_{-0.007}^{+0.007}$ & $2.0_{-1.2}^{+1.5}$ & 1.0 & $19.003_{-0.009}^{+0.010}$ & $5.2_{-3.8}^{+5.6}$ \\
\hline & - & -1.3 & -0.0 & $437_{-0.003}^{+0.003}$ & $1.7_{-0.8}^{+0.9}$ & 1.0 & $12.153_{-0.008}^{+0.006}$ & $2.2_{-1.2}^{+1.5}$ & 1.0 & $19.007_{-0.023}^{+0.014}$ & $8.2_{-5.7}^{+7.4}$ \\
\hline & - & - & - & $8.439_{-}^{+}$ & $1.7_{-0.8}^{+0.9}$ & 1.0 & $12.149_{-0.008}^{+0.008}$ & $2.4_{-1.3}^{+2.0}$ & 1.0 & $19.000_{-0.026}^{+0.014}$ & $\begin{array}{l}5.1_{-4.1}^{+6.0} \\
+6.0\end{array}$ \\
\hline & $185_{-0.006}^{+0.008}$ & $1.5_{-1.1}^{+1.1}$ & 1.0 & $8.439_{-}^{+}$ & $1.9_{-0.8}^{+0.9}$ & 1.0 & - & - & - & $19.001_{-0.011}^{+0.010}$ & $8.1_{-}^{+}$ \\
\hline & & $1.6_{-1.0}^{+1.1}$ & 1.0 & $8.436_{-0.008}^{+0.008}$ & $3.4_{-1.4}^{+0.6}$ & $13.6_{-6.8}^{+8.2}$ & - & - & - & $19.000_{-0.011}^{+0.010}$ & $8.1_{-4.9}^{+6.7}$ \\
\hline $40 \quad 0.975$ & $6.185_{-0.004}^{+0.004}$ & $2.2_{-1.1}^{+1.0}$ & 1.0 & $8.436_{-0.008}^{+0.008}$ & $3.4_{-1.4}^{+1.4}$ & $13.6_{-6.8}^{+8.2 \mathrm{~b}}$ & - & - & - & $18.996_{-0.016}^{+0.021}$ & $7.5_{-5.3}^{+7.6}$ \\
\hline
\end{tabular}

a $\lambda$ is in units of $\AA$, Flux is in units of $10^{-5}$ photons $\mathrm{s}^{-1} \mathrm{~cm}^{-2}$, and $\sigma$ is in units of $10^{-3} \AA$.

a $67 \%$ confidence values 
Table 4

Intercombination lines along the phases for $4 \mathrm{U} 1822-371^{\mathrm{a}}$

\begin{tabular}{|c|c|c|c|c|c|c|c|c|c|}
\hline \multirow[t]{2}{*}{ phase } & \multicolumn{3}{|c|}{ MgXI i } & \multicolumn{3}{|c|}{ NeIXi } & \multicolumn{3}{|c|}{ OVII i } \\
\hline & $\lambda$ & Flux & $\sigma$ & $\lambda$ & Flux & $\sigma$ & $\lambda$ & Flux & $\sigma$ \\
\hline 10.0 & $9.243_{-0.008}^{+0.006}$ & $1.5_{-11}^{+1.3}$ & 1.0 & - & - & - & $21.830_{-0.009}^{+0.009}$ & $21.3_{-13.7}^{+22.1}$ & 1.0 \\
\hline 20.025 & $9.241_{-0.013}^{+0.008}$ & $1.6_{-1.1}^{+1.4}$ & $<13.1$ & $13.549_{-0.011}^{+0.016}$ & $6.5_{-3.2}^{+3.4}$ & $17.7_{-9.7}^{+14.1}$ & $21.826_{-0.009}^{+0.011}$ & $11.7_{-8.3}^{+12.4}$ & 1.0 \\
\hline 30.05 & $9.243_{-0.008}^{+0.005}$ & $2.4_{-1.1}^{+1.7}$ & $<10.1$ & $13.557_{-0.011}^{+0.012}$ & $5.8_{-2.6}^{+3.6}$ & $12.9_{-7.1}^{+13.6}$ & $21.835_{-0.007}^{+0.006}$ & $18.4_{-10.1}^{+14.2}$ & 1.0 \\
\hline 40.075 & $9.233_{-0.010}^{+0.011}$ & $4.1_{-1.9}^{+2.0}$ & $16.7_{-6.8}^{+11.7}$ & $13.561_{-0.008}^{+0.007}$ & $7.1_{-2.8}^{+3.3}$ & $11.4_{-4.6}^{+5.1} \mathrm{~b}$ & $21.836_{-0.006}^{+0.009}$ & $18.8_{-10.1}^{+14.1}$ & 1.0 \\
\hline 50.1 & $9.241_{-0.016}^{+0.005}$ & $2.7_{-1.2}^{+2.3}$ & $<10.6$ & $13.565_{-0.008}^{+0.007}$ & $8.3_{-3.1}^{+3.6}$ & $12.9_{-6.9}^{+8.9}$ & $21.837_{-0.007}^{+0.009}$ & $15.4_{-9.2}^{+13.5}$ & 1.0 \\
\hline 60.125 & $9.232_{-0.007}^{+0.006}$ & $5.6_{-1.8}^{+1.0}$ & $15.4_{-5.6}^{+7.0}$ & $13.565_{-0.005}^{+0.005}$ & $9.7_{-3.2}^{+3.6}$ & $\begin{array}{l}9.5_{-6.1}^{+7.3} \\
\end{array}$ & $21.825_{-0.015}^{+0.016}$ & $37.4_{-17.3}^{+20.9}$ & $25.2_{-7.9}^{+8.7 \mathrm{~b}}$ \\
\hline 70.15 & $9.234_{-0.004}^{+0.009}$ & $5.2_{-2.2}^{+2.0}$ & $12.7_{-8.8}^{+6.3}$ & $13.564_{-0.005}^{+0.004}$ & $13.8_{-3.8}^{+4.4}$ & $9.9_{-7.0}^{+7.1}$ & $21.824_{-0.013}^{+0.015}$ & $42.3_{-17.5}^{+21.7}$ & $24.5_{-11.5}^{+14.7}$ \\
\hline 80.175 & $9.238_{-0.004}^{+0.003}$ & $5.0_{-1.5}^{+1.7}$ & $7.7_{-4.4}^{+5.2}$ & $13.562_{-0.005}^{+0.005}$ & $15.0_{-3.8}^{+4.8}$ & $14.0_{-4.7}^{+6.2}$ & $21.814_{-0.011}^{+0.011}$ & $61.3_{-20.7}^{+24.6}$ & $26.6_{-7.7}^{+12.4}$ \\
\hline 90.2 & $9.236_{-0.004}^{+0.005}$ & $4.4_{-1.5}^{+1.7}$ & $\begin{array}{r}7.9_{-7.8}^{+5.9} \\
\end{array}$ & $13.560_{-0.006}^{+0.006}$ & $15.5_{-4.1}^{+4.8}$ & $17.4_{-6.3}^{+8.1}$ & $21.808_{-0.011}^{+0.010}$ & $62.5_{-20.7}^{+24.8}$ & $25.1_{-7.7}^{+13.0}$ \\
\hline 100.225 & $9.236_{-0.005}^{+0.005}$ & $4.6_{-1.6}^{+1.7}$ & $8.0_{-3.7}^{+3.6 \mathrm{~b}}$ & $13.557_{-0.008}^{+0.009}$ & $15.9_{-4.5}^{+5.1}$ & $24.1_{-7.5}^{+11.2}$ & $21.796_{-0.014}^{+0.011}$ & $63.6_{-21.9}^{+26.0}$ & $29.5_{-10.4}^{+16.2}$ \\
\hline 110.25 & $9.232_{-0.007}^{+0.005}$ & $4.3_{-1.8}^{+2.1}$ & $7.8_{-5.6}^{+6.3 \mathrm{~b}}$ & $13.551_{-0.006}^{+0.006}$ & $18.1_{-4.6}^{+5.2}$ & $18.5_{-6.9}^{+9.1}$ & $21.793_{-0.012}^{+0.011}$ & $65.3_{-21.9}^{+25.9}$ & $27.4_{-9.0}^{+12.9}$ \\
\hline 120.275 & $9.230_{-0.005}^{+0.005}$ & $4.3_{-1.6}^{+2.0}$ & $7.8_{-6.7}^{+9.1}$ & $13.549_{-0.005}^{+0.005}$ & $17.8_{-4.1}^{+4.8}$ & $15.2_{-4.8}^{+7.9}$ & $21.789_{-0.016}^{+0.013}$ & $51.2_{-20.2}^{+24.7}$ & $25.2_{-10.9}^{+18.0}$ \\
\hline 130.3 & $9.228_{-0.006}^{+0.005}$ & $5.8_{-2.0}^{+2.4}$ & $12.4_{-6.2}^{+10.0}$ & $13.544_{-0.006}^{+0.006}$ & $15.5_{-4.2}^{+5.6}$ & $15.9_{-6.4}^{+12.7}$ & $21.800_{-0.032}^{+0.009}$ & $49.4_{-24.4}^{+28.0}$ & $30.5_{-18.1}^{+24.5}$ \\
\hline 140.325 & $9.225_{-0.006}^{+0.006}$ & $6.9_{-2.1}^{+2.5}$ & $16.1_{-5.4}^{+9.0}$ & $13.541_{-0.007}^{+0.007}$ & $13.9_{-4.2}^{+4.8}$ & $18.0_{-7.4}^{+9.4}$ & $21.798_{-0.020}^{+0.018}$ & $37.7_{-19.0}^{+23.6}$ & $28.0_{-15.9}^{+21.4}$ \\
\hline 150.35 & $9.222_{-0.006}^{+0.006}$ & $7.4_{-2.2}^{+2.7}$ & $15.5_{-5.9}^{+9.4}$ & $13.537_{-0.007}^{+0.007}$ & $16.0_{-4.4}^{+4.2}$ & $20.5_{-6.7}^{+9.4}$ & $21.800_{-0.021}^{+0.019}$ & $37.8_{-18.5}^{+23.4}$ & $30.4_{-13.6}^{+22.5}$ \\
\hline 160.375 & $9.221_{-0.006}^{+0.006}$ & $7.9_{-2.3}^{+2.8}$ & $16.9_{-6.0}^{+8.6}$ & $13.545_{-0.009}^{+0.008}$ & $18.3_{-5.1}^{+6.0}$ & $26.7_{-8.5}^{+13.2}$ & $21.806_{-0.015}^{+0.015}$ & $37.3_{-17.5}^{+21.8}$ & $23.6_{-11.0}^{+15.0}$ \\
\hline 170.4 & $9.223_{-0.006}^{+0.007}$ & $6.8_{-2.2}^{+2.6}$ & $\begin{array}{l}15.3_{-6.0}^{+8.0} \\
\end{array}$ & $13.545_{-0.009}^{+0.008}$ & $15.8_{-4.6}^{+5.1}$ & $22.9_{-7.7}^{+11.9}$ & $21.813_{-0.014}^{+0.015}$ & $30.0_{-15.1}^{+19.3}$ & $20.3_{-10.8}^{+13.0}$ \\
\hline 180.425 & $9.224_{-0.009}^{+0.008}$ & $7.6_{-2.6}^{+3.2}$ & $21.3_{-7.7}^{+11.9}$ & $13.535_{-0.013}^{+0.011}$ & $13.9_{-4.9}^{+7.0}$ & $27.3_{-10.7}^{+25.3}$ & $21.818_{-0.015}^{+0.015}$ & $26.1_{-14.0}^{+18.1}$ & $18.9_{-10.7}^{+10.8}$ \\
\hline 190.45 & $9.225_{-0.010}^{+0.010}$ & $7.5_{-2.6}^{+3.2}$ & $24.6_{-8.0}^{+12.2}$ & $13.540_{-0.010}^{+0.011}$ & $14.7_{-4.8}^{+6.0}$ & $27.4_{-9.0}^{+19.8}$ & $21.816_{-0.013}^{+0.013}$ & $26.5_{-14.0}^{+18.0}$ & $16.8_{-6.4}^{+6.7 \mathrm{~b}}$ \\
\hline 200.475 & $9.227_{-0.012}^{+0.010}$ & $6.3_{-2.6}^{+3.4}$ & $24.6_{-9.8}^{+1.0}$ & $13.540_{-0.010}^{+0.015}$ & $18.3_{-5.4}^{+6.5}$ & $33.3_{-10.1}^{+15.4}$ & $21.811_{-0.021}^{+0.020}$ & $21.1_{-14.2}^{+17.0}$ & $21.5_{-8.9}^{+10.4 \mathrm{~b}}$ \\
\hline 210.5 & $9.216_{-0.006}^{+0.010}$ & $1.8_{-1.1}^{+1.2}$ & 1.0 & $13.544_{-0.015}^{+0.011}$ & $16.1_{-5.1}^{+10.6}$ & $29.8_{-10.7}^{+33.8}$ & $21.801_{-0.051}^{+0.021}$ & $21.8_{-13.9}^{+18.2}$ & $23.3_{-19.5}^{+27.6}$ \\
\hline 220.525 & -0.000 & -1.1 & - & $13.548_{-0.009}^{+0.009}$ & $14.3_{-4.3}^{+5.1}$ & $24.3_{-7.0}^{+14.8}$ & $21.814_{-0.009}^{+0.008}$ & $12.4_{-8.4}^{+13.0}$ & 1.0 \\
\hline 230.55 & - & - & - & $13.550_{-0.012}^{+0.011}$ & $12.1_{-4.2}^{+5.3}$ & $24.9_{-8.4}^{+1.0 .7}$ & $21.819_{-0.008}^{+0.007}$ & $14.7_{-9.4}^{+14.0}$ & 1.0 \\
\hline 240.575 & - & - & - & $13.554_{-0.010}^{+0.010}$ & $11.7_{-4.1}^{+4.8}$ & $21.9_{-8.5}^{+13.2}$ & $21.818_{-0.007}^{+0.007}$ & $19.4_{-11.0}^{+15.7}$ & 1.0 \\
\hline 250.6 & - & - & - & $13.560_{-0.010}^{+0.010}$ & $9.7_{-3.7}^{+4.3}$ & $19.4_{-8.6}^{+13.7}$ & $21.809_{-0.020}^{+0.019}$ & $45.9_{-20.1}^{+24.3}$ & $35.8_{-12.7}^{+20.4}$ \\
\hline 260.625 & - & - & - & $13.562_{-0.012}^{+0.011}$ & $9.8_{-3.8}^{+4.1}$ & $22.4_{-10.2}^{+16.6}$ & $21.810_{-0.019}^{+0.019}$ & $\begin{array}{r}-20 . \frac{1}{1} \\
48.1_{-20.3}^{+24.3}\end{array}$ & $37.2_{-12.2}^{+19.7}$ \\
\hline 270.65 & - & - & - & $13.566_{-0.009}^{+0.008}$ & $9.0_{-3.4}^{+4.0}$ & $16.4_{-7.8}^{+11.6}$ & $21.818_{-0.023}^{+0.020}$ & $42.7_{-20.2}^{+24.0}$ & $33.9_{-16.9}^{+24.6}$ \\
\hline 280.675 & - & - & - & $13.565_{-0.011}^{+0.010}$ & $7.3_{-3.1}^{+3.7}$ & $17.0_{-9.0}^{+12.8}$ & $21.825_{-0.018}^{+0.014}$ & $39.2_{-17.9}^{+22.8}$ & $23.8_{-10.9}^{+19.7}$ \\
\hline 290.7 & - & - & - & $13.567_{-0.014}^{+0.011}$ & $7.4_{-3.1}^{+3.1 .6}$ & $18.4_{-8.7}^{+14.2}$ & $21.822_{-0.020}^{+0.015}$ & $34.2_{-16.6}^{+21.4}$ & $23.0_{-11.0}^{+21.9}$ \\
\hline 300.725 & $9.223_{-0.006}^{+0.007}$ & $1.2_{-0.9}^{+1.0}$ & 1.0 & $13.562_{-0.020}^{+0.014}$ & $7.8_{-3.5}^{+4.7}$ & $24.5_{-11.8}^{+21.9}$ & $21.826_{-0.016}^{+0.015}$ & $29.6_{-14.2}^{+18.5}$ & $23.1_{-9.8}^{+18.4}$ \\
\hline 310.75 & $9.224_{-0.006}^{+0.007}$ & $1.2_{-0.9}^{+1.0}$ & 1.0 & - & - & - & $21.850_{-0.009}^{+0.010}$ & $14.7_{-9.4}^{+14.0}$ & 1.0 \\
\hline $\begin{array}{ll}32 & 0.775\end{array}$ & -0.000 & -0.9 & - & - & - & - & -0.009 & - & - \\
\hline $\begin{array}{lll}33 & 0.8\end{array}$ & - & & - & - & - & - & - & - & - \\
\hline 340.825 & $9.229_{-0.007}^{+0.007}$ & $1.0_{-0.8}^{+0.9}$ & 1.0 & - & - & - & - & - & - \\
\hline 350.85 & $9.228_{-0.004}^{+0.006}$ & $1.3_{-0.8}^{+0.9}$ & 1.0 & - & - & - & - & - & - \\
\hline 360.875 & $9.228_{-0.004}^{+0.005}$ & $\begin{array}{l}1.3_{-0.8}^{+0.8} \\
+0.8\end{array}$ & 1.0 & $13.561_{-0.006}^{+0.005}$ & $3.4_{-1.8}^{+2.2}$ & 1.0 & $21.817_{-0.020}^{+0.020}$ & $33.1_{-15.4}^{+19.4}$ & $34.7_{-12.3}^{+19.5}$ \\
\hline 370.9 & $9.229_{-0.005}^{+0.006}$ & $\begin{array}{l}1.1_{-0.8}^{+0.9} \\
\end{array}$ & 1.0 & $13.566_{-0.011}^{+0.013}$ & $6.3_{-3.1}^{+3.7}$ & $15.8_{-14.1}^{+15.2}$ & $21.820_{-0.030}^{+0.030}$ & $22.1_{-14.8}^{+19.3}$ & $30.6_{-10.7}^{+15.9 b}$ \\
\hline 380.925 & $9.231_{-0.005}^{+0.004}$ & $1.6_{-0.9}^{+0.0}$ & 1.0 & $13.560_{-0.011}^{+0.012}$ & $4.7_{-2.9}^{+8.3}$ & $<34.7$ & $21.822_{-0.025}^{+0.024}$ & $20.4_{-13.4}^{+17.0}$ & $24.3_{-8.1}^{+12.0 \mathrm{~b}}$ \\
\hline 390.95 & $9.231_{-0.006}^{+0.005}$ & $1.4_{-0.8}^{+0.9}$ & 1.0 & $13.560_{-0.051}^{+0.014}$ & $4.5_{-2.9}^{+10.2}$ & $14.4_{-11.8}^{+14.4 \mathrm{~b}}$ & $21.831_{-0.018}^{+0.016}$ & $26.0_{-13.9}^{+18.4}$ & $21.5_{-10.1}^{+18.0}$ \\
\hline $40 \quad 0.975$ & $9.235_{-0.010}^{+0.009}$ & $1.9_{-1.2}^{+1.3}$ & $9.6_{-4.3}^{+5.0 \mathrm{~b}}$ & $13.560_{-0.051}^{+0.014}$ & $4.5_{-2.9}^{+10.2}$ & $14.4_{-9.8}^{+9.5 \mathrm{~b}}$ & $21.832_{-0.016}^{+0.014}$ & $24.2_{-13.2}^{+1.3 .9}$ & $17.5_{-10.6}^{+10.1}$ \\
\hline
\end{tabular}

a $\lambda$ is in units of $\AA$, Flux is in units of $10^{-5}$ photons $\mathrm{s}^{-1} \mathrm{~cm}^{-2}$, and $\sigma$ is in units of $10^{-3} \AA$.

a $67 \%$ confidence levels 
Table 5

Fe lines along the phases for $4 \mathrm{U} 1822-371^{\mathrm{a}}$

\begin{tabular}{|c|c|c|c|c|c|c|}
\hline \multirow[t]{2}{*}{ phase } & \multicolumn{3}{|c|}{$\mathrm{FeK} \alpha$} & \multicolumn{3}{|c|}{ FeXXVI Ly $\alpha$} \\
\hline & $\lambda$ & Flux & $\sigma$ & $\lambda$ & Flux & $\sigma$ \\
\hline 1(upper) & $1.937_{-0.001}^{+0.001}$ & $46.7_{-8.0}^{+10.0}$ & $5.7_{-2.2}^{+2.5}$ & $1.782_{-0.003}^{+0.003}$ & $20.8_{-8.0}^{+10.0}$ & $<7.1$ \\
\hline 2(decreasing) & $1.944_{-0.009}^{+0.008}$ & $12.2_{-10.0}^{+10.6}$ & 1.0 & $1.786_{-0.004}^{+0.002}$ & $10.2_{-10 .}^{+11.6}$ & 1.0 \\
\hline 3(bottom) & $1.938_{-0.003}^{+0.003}$ & $16.8_{-4.8}^{+6.4}$ & $<7.2$ & $1.786_{-0.005}^{+0.006}$ & $7.4_{-4.9}^{+4.9}$ & 1.0 \\
\hline 4(eclipse) & $1.936_{-0.003}^{+0.007}$ & $14.9_{-7.5}^{+7.5}$ & 1.0 & -0.000 & -4.9 & - \\
\hline 5 (rising) & $1.937_{-0.002}^{+0.002}$ & $31.6_{-7.9}^{+8.3}$ & $<5.7$ & $1.783_{-0.004}^{+0.003}$ & $19.3_{-7.3}^{+7.3}$ & 1.0 \\
\hline
\end{tabular}

$\overline{\text { a } \lambda \text { is in units of } \AA \text {, Flux is in units of } 10^{-5} \text { photons } \mathrm{s}^{-1} \mathrm{~cm}^{-2} \text {, and } \sigma \text { is in }}$ units of $10^{-3} \AA$.

Table 6

Ne IX triplet line ratios along the phases for 4U1822-371

\begin{tabular}{l|ccc|cc|c}
\hline \hline phase & $\begin{array}{c}\lambda^{\mathrm{a}} \\
(\AA)\end{array}$ & $\begin{array}{c}\sigma \\
\left(10^{-3} \AA\right)\end{array}$ & $\left.\begin{array}{c}\mathrm{G} \\
\left(10^{-5} \text { photons } \mathrm{s}^{-1}\right.\end{array} \mathrm{cm}^{-2}\right)$ & $\begin{array}{c}\mathrm{G} \\
(\mathrm{f}+\mathrm{i}) / \mathrm{r}\end{array}$ & $\begin{array}{c}\text { Cash/dof } \\
\mathrm{f} / \mathrm{i}\end{array}$ & \\
\hline 1(upper) & $13.541_{-0.005}^{+0.007}$ & $24_{-5}^{+7}$ & $15.4_{-3.2}^{+3.4}$ & $4.8_{-2.0}^{+7.8}$ & $<0.3$ & $74 / 81$ \\
2(decreasing) & $13.560_{-0.020}^{+0.009}$ & $18_{-9}^{+16}$ & $9.2_{-4.7}^{+5.6}$ & - & - & $67 / 81$ \\
3(bottom) & $13.565_{-0.009}^{+0.009}$ & $20_{-7}^{+10}$ & $6.6_{-2.1}^{+2.6}$ & $>3.2$ & $<0.4$ & $111 / 81$ \\
4(eclipse) & $13.470_{-0.005}^{+0.016}$ & 1.0 & $3.1_{-2.1}^{+2.7}$ & - & - & $77 / 81$ \\
5(rising) & $13.563_{-0.004}^{+0.004}$ & $14_{-5}^{+7}$ & $14.4_{-3.3}^{+3.9}$ & $4.9_{-2.3}^{+10.2}$ & $<0.2$ & $65 / 81$ \\
& & & & & & \\
\hline
\end{tabular}

a strongest line in the triplet complex: intercombination line for phase 1,2,3,5; resonance line for phase 4.

Table 7

Triplet line ratios for two new observations of $4 \mathrm{U} 1822-371$

\begin{tabular}{l|ccc|cc|c}
\hline \hline Ion & $\begin{array}{c}\lambda(i) \\
(\AA)\end{array}$ & $\begin{array}{c}\sigma \\
\left(10^{-3} \AA\right)\end{array}$ & $\left.\begin{array}{c}\text { flux } \\
\left(10^{-5} \text { photons s }\right.\end{array} \mathrm{cm}^{-2}\right)$ & $\begin{array}{c}\mathrm{G} \\
(\mathrm{f}+\mathrm{i}) / \mathrm{r}\end{array}$ & $\begin{array}{c}\mathrm{R} \\
\mathrm{f} / \mathrm{i}\end{array}$ & Cash/dof \\
\hline Mg XI & $9.232_{-0.004}^{+0.005}$ & $17_{-4}^{+5}$ & $3.9_{-0.8}^{+1.0}$ & $>3.8$ & $<0.2$ & $16 / 27$ \\
Ne IX & $13.553_{-0.003}^{+0.007}$ & $24_{-4}^{+5}$ & $11.0_{-1.6}^{+1.7}$ & $5.5_{-2.0}^{+6.0}$ & $<0.2$ & $89 / 81$ \\
O VII & $21.817_{-0.013}^{+0.005}$ & $36_{-8}^{+10}$ & $38.4_{-7.6}^{+8.7}$ & $4.5_{-1.8}^{+5.5}$ & $<0.09$ & $111 / 81$ \\
& & & & & & \\
\hline
\end{tabular}



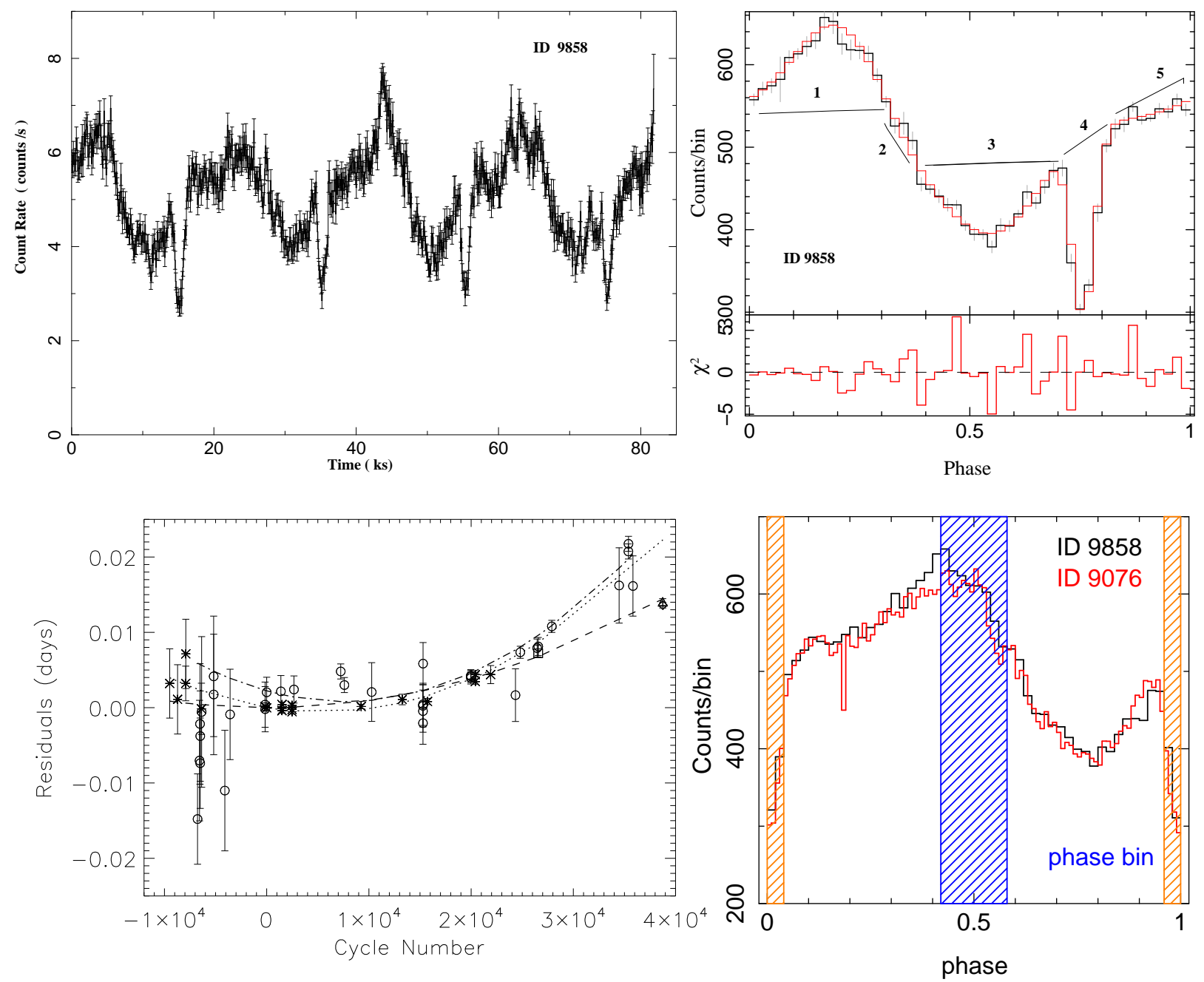

Figure 1. Upper left - Light curve for ID 9858 at wavelengths $\lambda>1.5 \AA$; Upper right — The fitted folded phase light curve of ID9858: black - observational curve; red - fitted curve. Short black lines and index mark the phase bins for Fe lines analysis. Lower left - The 4U1822-371 partial eclipse timing residuals with respect to the best-fit linear ephemeris: previous X-ray eclipse measurements are at 68\% confidence levels - asterisk (Parmar et al. 2000, and reference therein); previous UV/Optical eclipse measurements at 90\% confidence - circle; new measurements at 90\% confidence( square - ID 671; triangle — ID9076; diamond — ID9858). The best quadratic ephemeris from our measurement (dash line) is indicated: dotted line - Parmar et al. (2000); dash-dotted line - UV/Optical fitting. Lower right - Phase folded light curves of ID9858 (black) \& ID9076 (red) with example out of eclipse phase bin width shown by blue shadows, and the in eclipse phase bin shown by the orange shadows. 

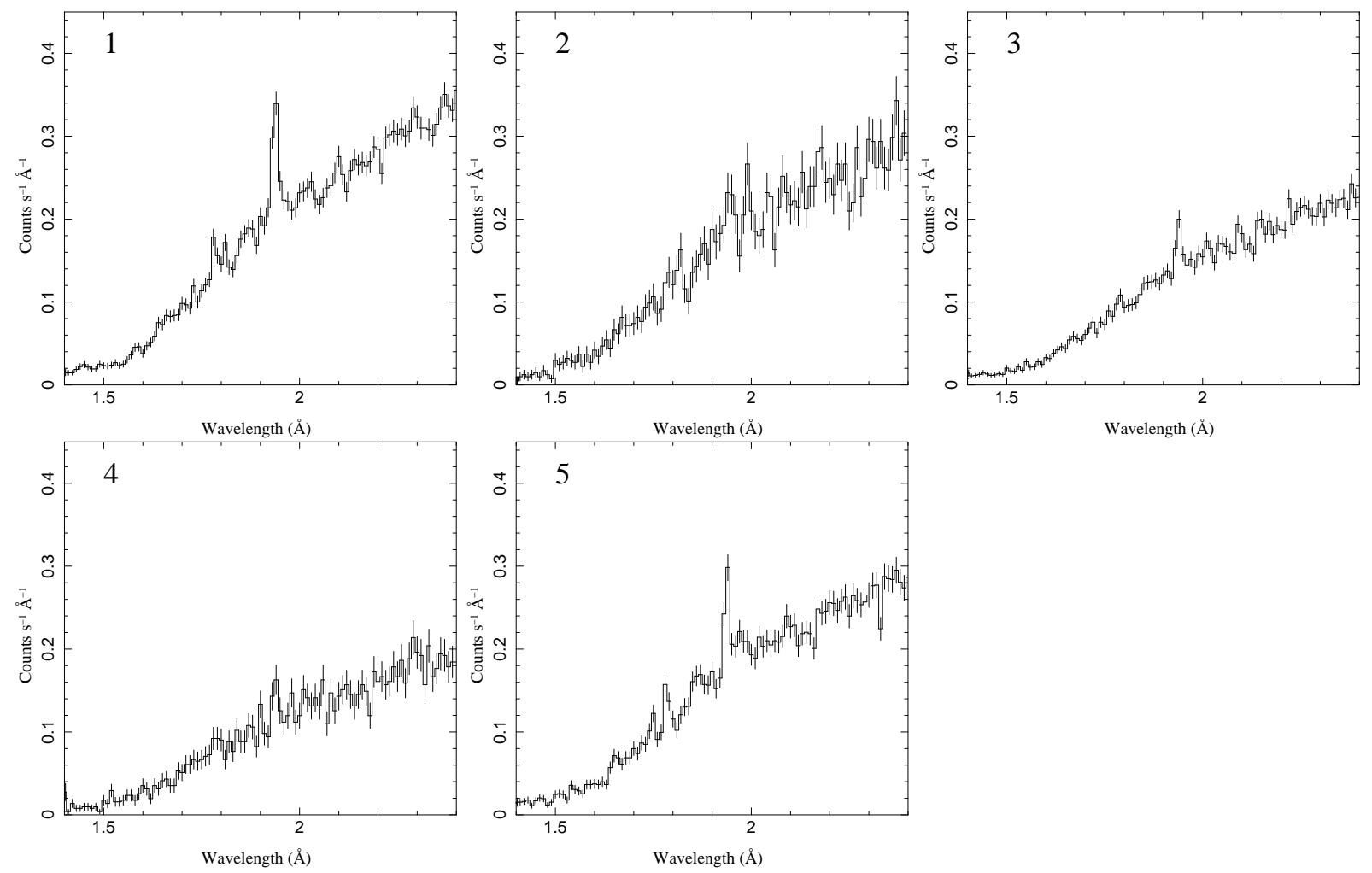

Figure 2. The phase folded spectra for the five phase bins shown in Figure 1
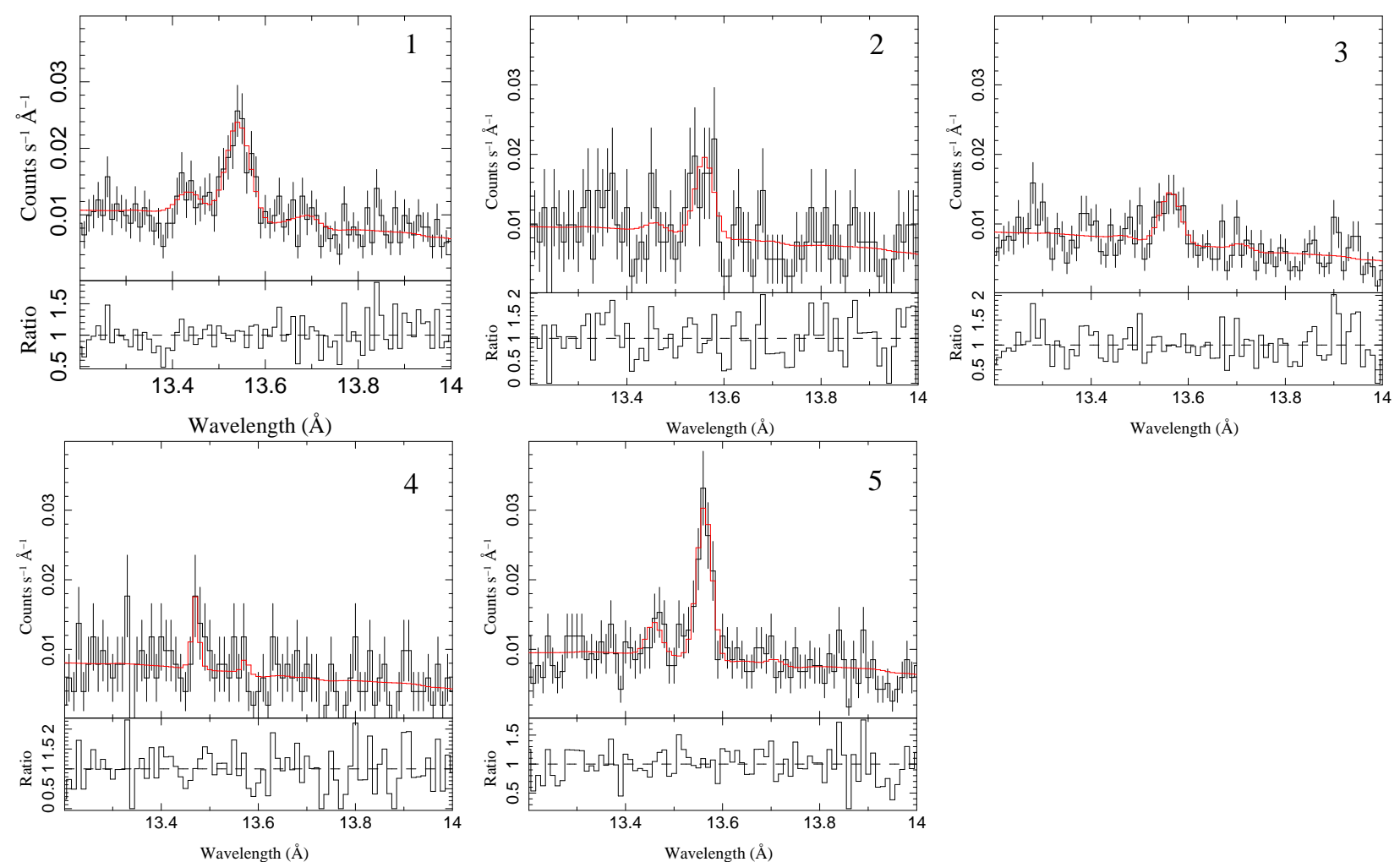

Figure 3. Ne IX triplet line spectra in the five phase bins shown in Figure 1 
Ji et al.
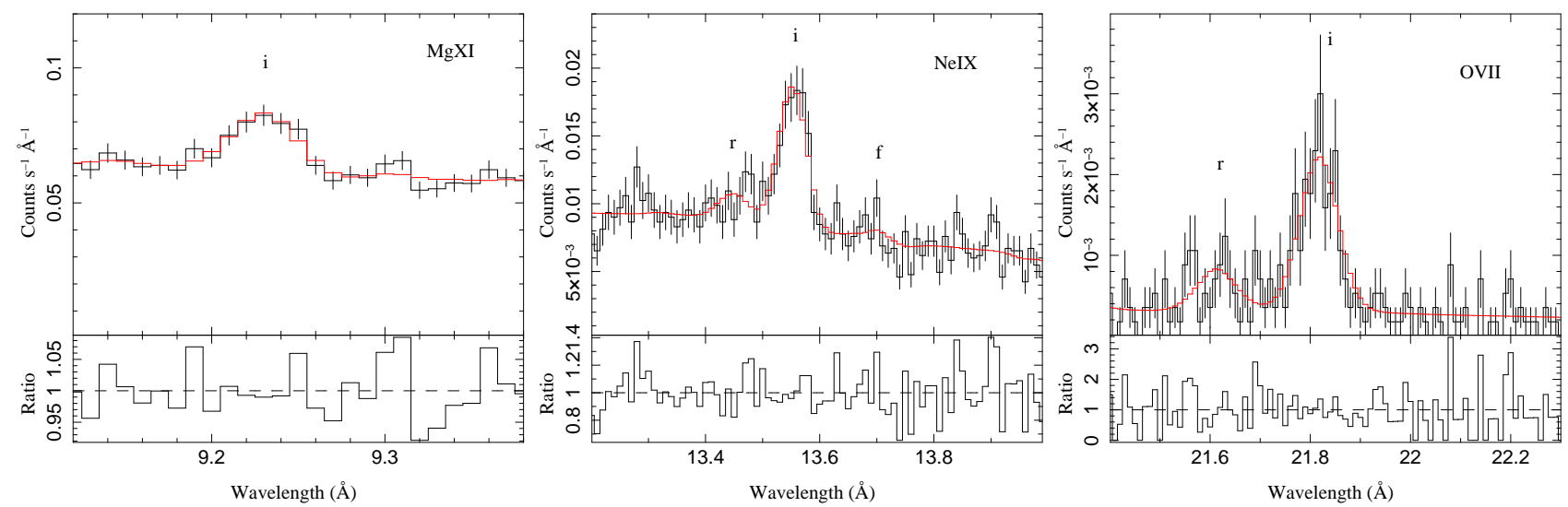

Figure 4. Triplet line spectra Mg XI (left), Ne IX (middle) and O VII (right) for the summed spectra from Obs IDs 9076 and 9858 . (See Table 4) 

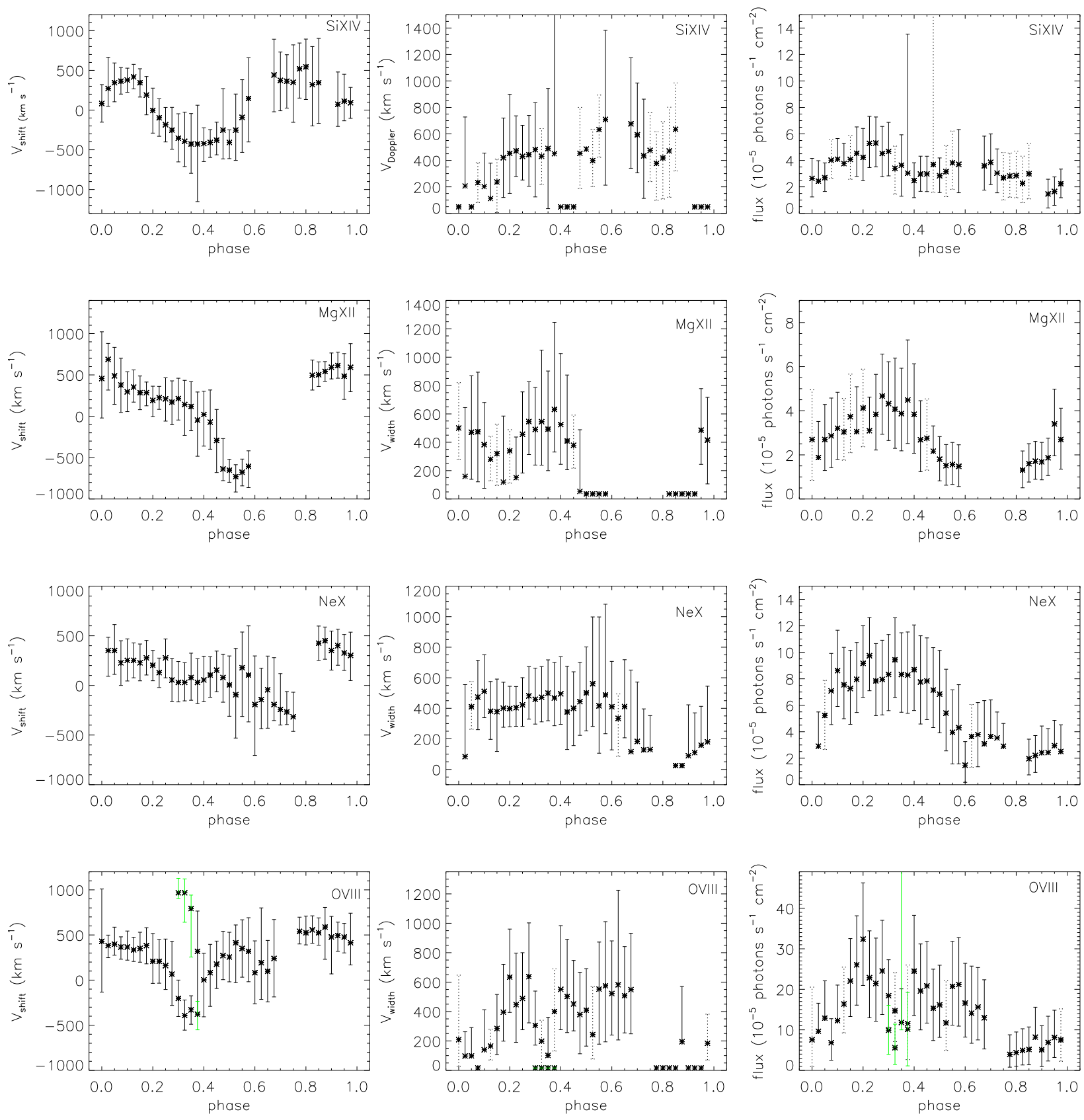

Figure 5. Line red-shift (left), broadening (middle), and flux (right) as a function of phase for Ly $\alpha$ lines of Si XIV, Mg XII, Ne X, and O VIII with $3 \sigma$ (solid line) and $1 \sigma$ (dotted line) error bars shown. Line broadening is fixed at $0.001 \AA$ for those points without error bars. Green lines mark additional detected line components. 

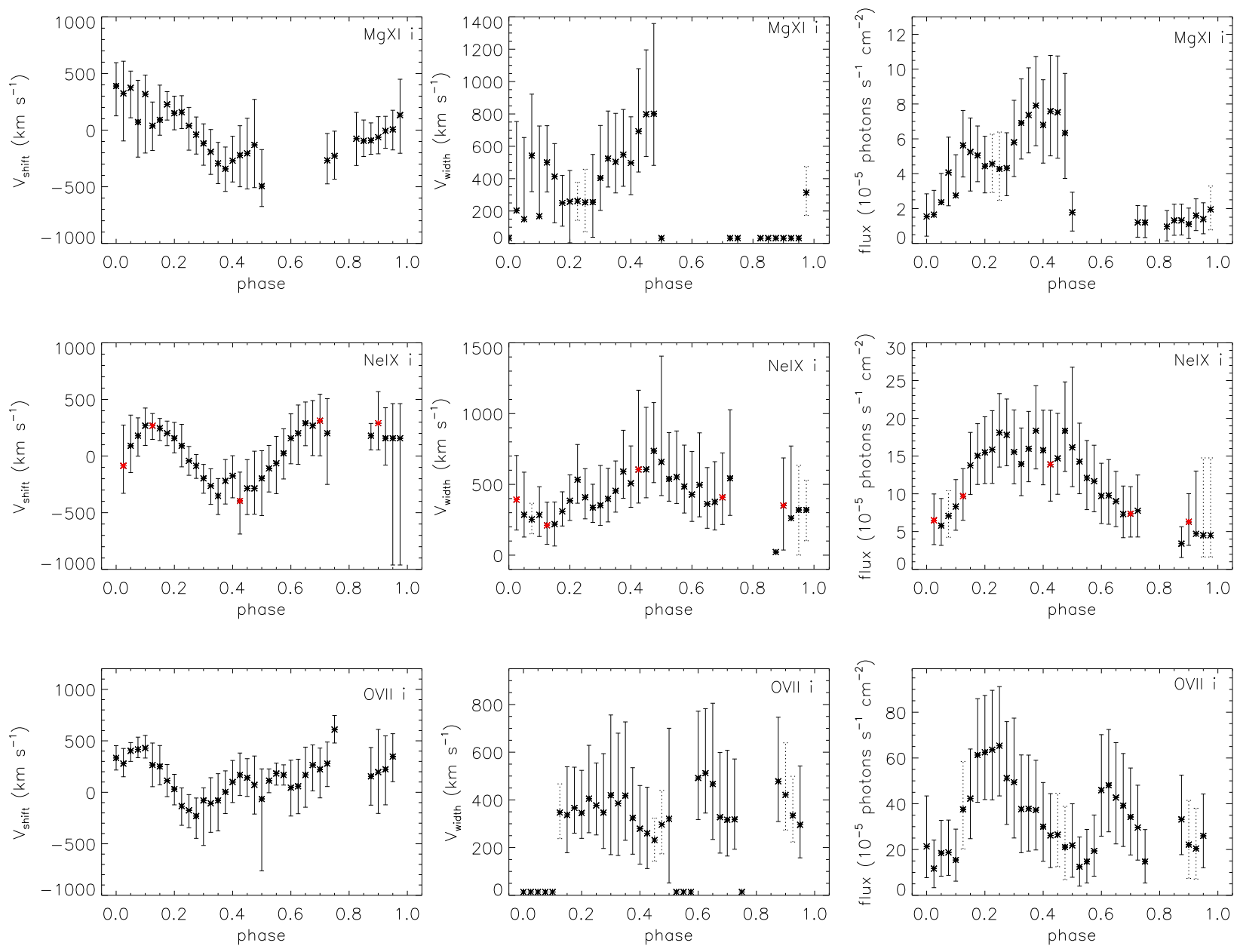

Figure 6. Line redshift (left), broadening (middle), and flux (right) as a function of phase for the intercombination lines of Mg XI (upper), Ne IX (middle), and O VII (lower), with $3 \sigma$ (solid line) and $1 \sigma$ (dotted line) error bars shown, respectively. Line broadening is fixed at $0.001 \AA$ for those points without error bars. Red points mark those phases where the spectra are shown in Figure 7 

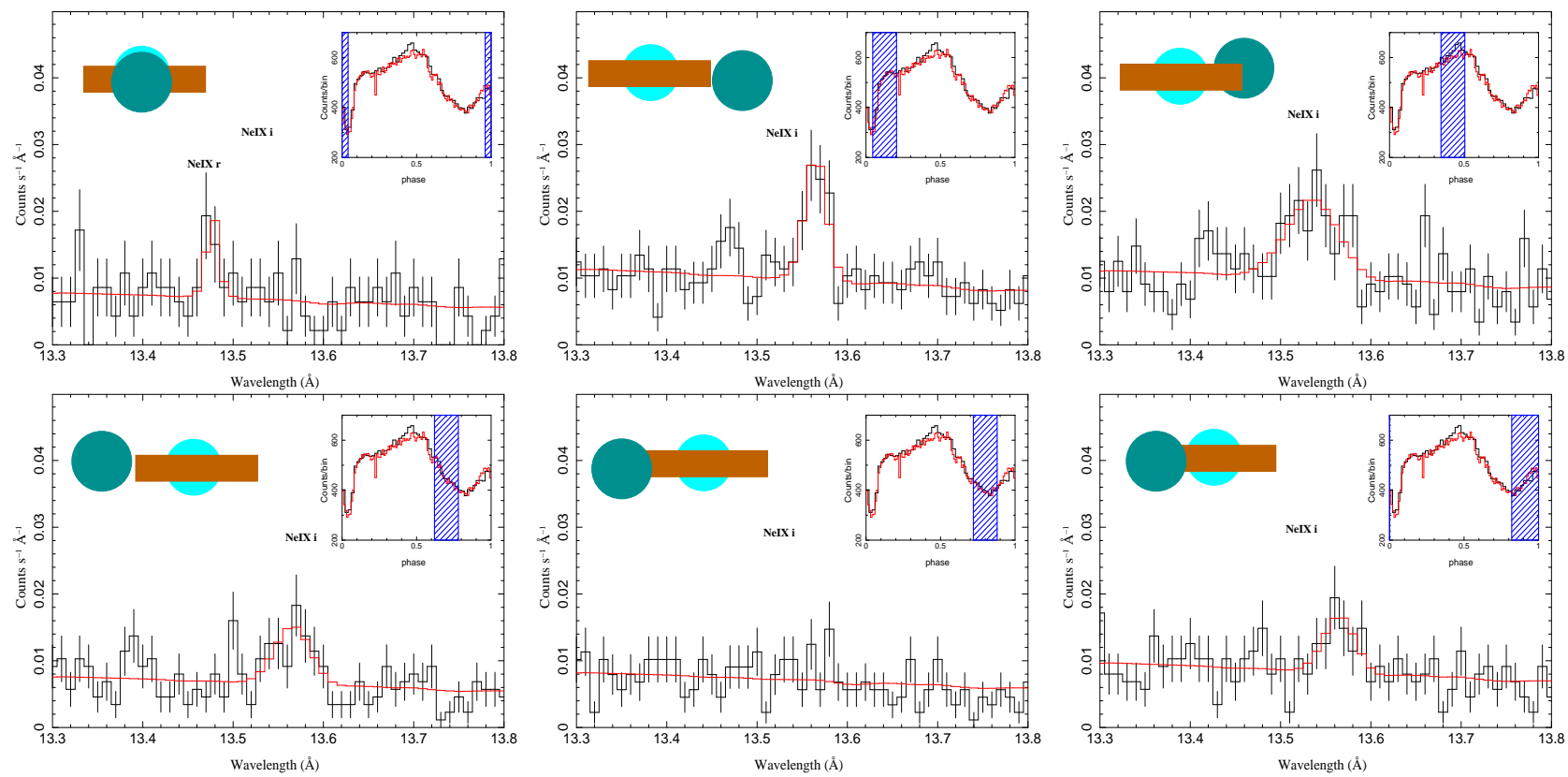

Figure 7. Variations of Ne IX intercombination line at the phases chosen in Figure 6 including the spectra without line detection for eclipse (left upper) and phase centered at 0.825 (middle lower). The pink lines mark the zero-velocity line location. The cartoon shows the hypothesized structure of the system (based upon the geometrical model of Heinz \& Nowak 2001). Each spectrum represents the average for the phase bin outlined by the blue shadow on the light curve in the right corner of each figure.
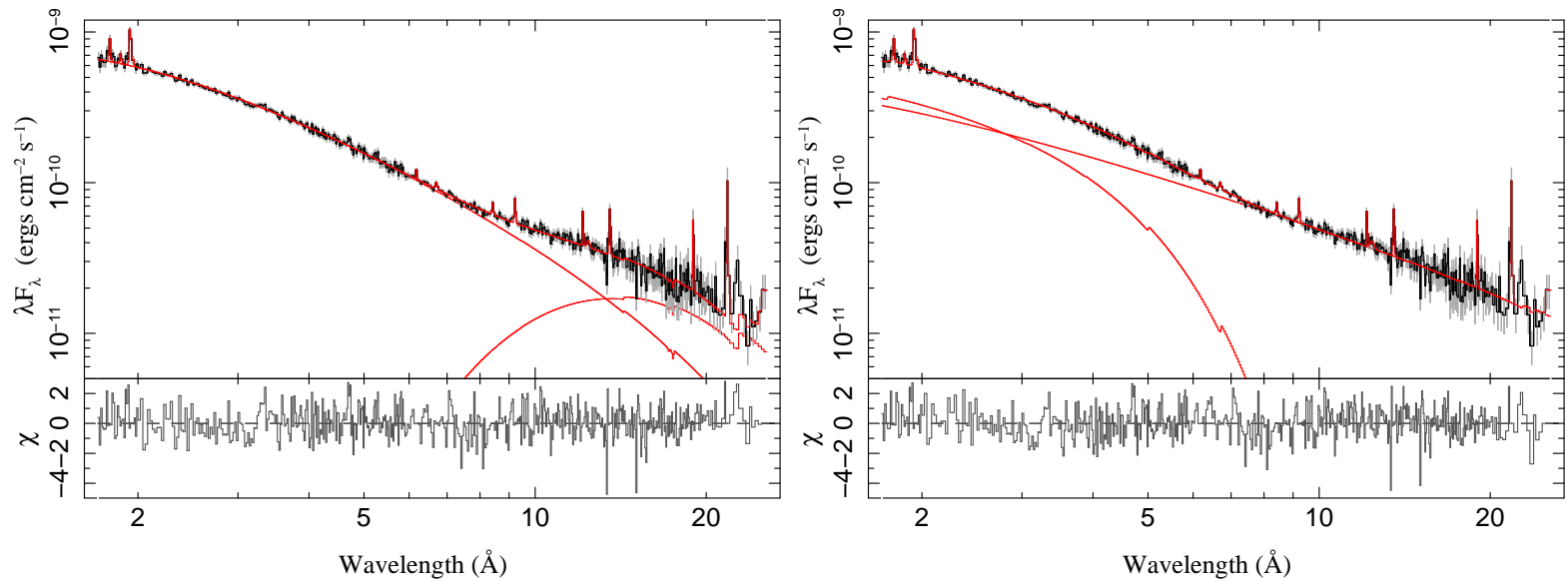

Figure 8. Continuum fits to the X-ray spectrum in phase 1. The left panel shows the fit with an exponentially cutoff power law plus a soft blackbody and high photo-electric absorption. The power law and black body components are individually shown. The right panel shows an alternative solution consisting of a single power law (the cutoff moves beyond the upper energy cutoff of the data) plus highly absorbed, partially covered power law with a partial covering fraction of $50 \%$. The overall, ISM contribution to the photo-electric absorption is low. The power law and covered power law are individually shown. 
סे.

${ }_{13.3}$

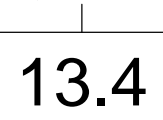

13.5

13.6

Wavelength ( $)$

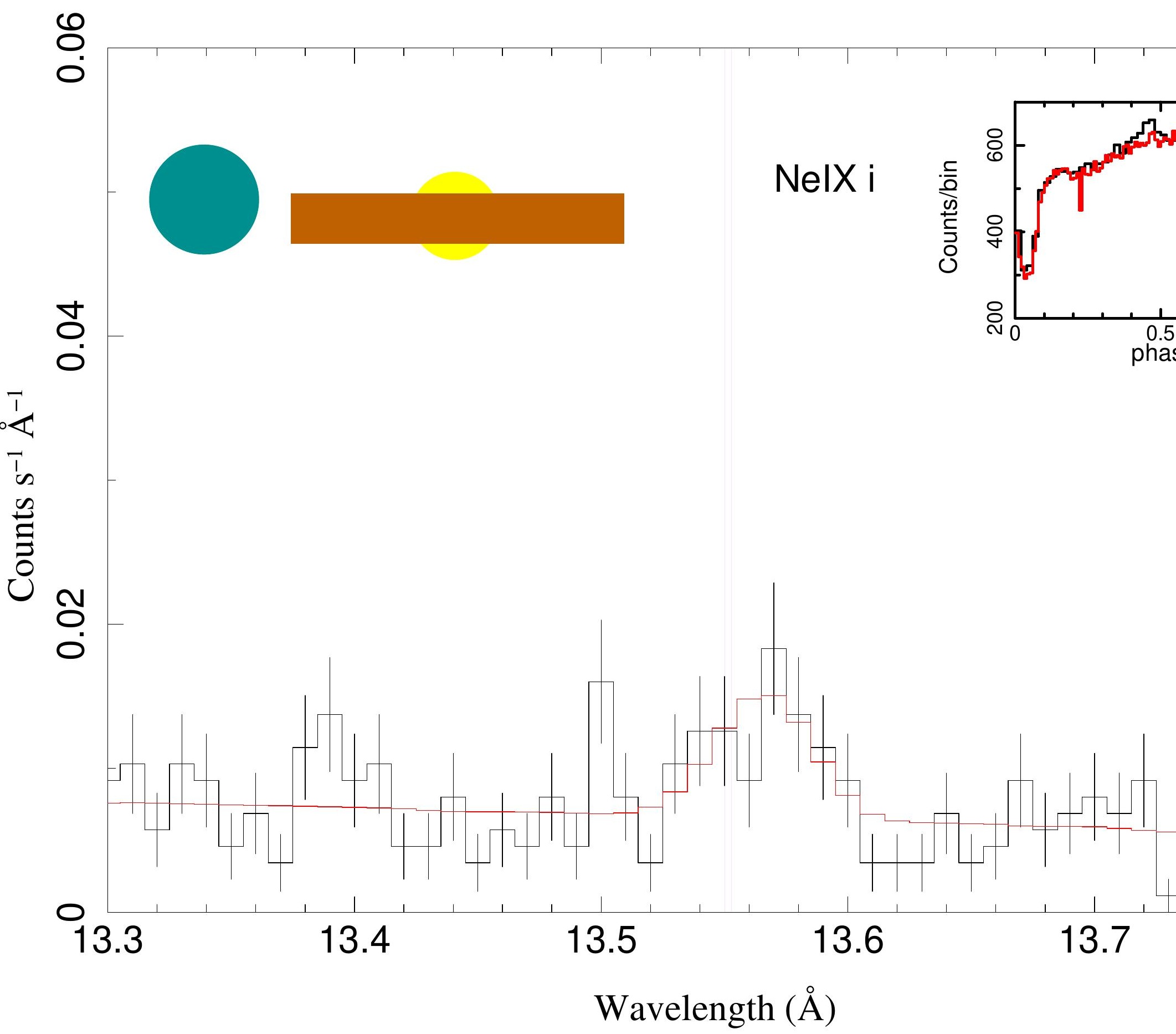

